\title{
Sealed Bid Auctions with Ambiguity: Theory and Experiments*
}

\author{
Yan Chen
}

\author{
Peter Katuscak
}

July 11,2003

\begin{abstract}
This study presents a theoretical model and laboratory experiment of the first and second price sealed bid auctions with independent private values, where the distribution of bidder valuations is unknown. We derive the symmetric equilibrium using the $\alpha$-MEU framework. We then test the theoretical predictions in the laboratory. In our experimental setting, ambiguity aversion is rejected in favor of ambiguity loving. Our results suggest that decision makers' ambiguity attitudes are context dependent. Another departure from previous experimental studies is the use of subjects as auctioneers. We find that compared to zero reserve prices the presence of auctioneers significantly reduces revenue in first price auctions. It also significantly reduces bidder earnings and efficiency. Without knowledge of the distribution of bidder valuations and with auctioneers, the first and second price auctions generate the same amount of revenue.
\end{abstract}

Keywords: sealed bid auctions, ambiguity, experiment JEL Classification: C91, D44, D83

${ }^{*}$ We thank Colin Camerer, David Cooper, Drew Fudenberg, Anthony Kwasnica, Dan Levin, Mark Machina, Rajiv Sarin, Katerina Sherstyuk, Robert Slonim, and seminar participants at Case Western, Harvard, Michigan, Ohio State, UC-San Diego, the 2002 American Economic Association meetings (Atlanta, GA), 2002 Economic Science Association meetings (Boston, MA), 2003 NBER/NSF Decentralization Conference (West Lafayette, IN) and North America Summer Econometric Society Meetings (Evanston, IL) for helpful comments. We thank Brian Chan, David Edelman and Casey Hui for excellent research assistance, and Jim Leady for programming for the experiment. Chen gratefully acknowledges the financial support from the National Science Foundation through grant no. SES-0079001. Any remaining errors are our own. Chen: School of Information, University of Michigan, 1075 Beal Avenue, Ann Arbor, MI 48109-2112. Katuscak and Ozdenoren: Department of Economics, The University of Michigan, 611 Tappan Street, Ann Arbor, MI 48109-1220. Email: yanchen@umich.edu, pkatus@umich.edu, emreo@umich.edu 


\section{Introduction}

Theoretical and experimental auction literature often assumes that both bidders and auctioneers know the distribution of bidder valuations. ${ }^{1}$ Consequently, nearly all of the results derive from such assumptions. However, in many real-world auctions, it is inappropriate to assume that bidders know the distribution from which opponent valuations are drawn. One prominent example is the Internet auctions. The online auction has become a fascinating and fast-growing exchange mechanism (Lucking-Reiley (2000a)). Online auction technology introduces several interesting features not available to traditional auctions. For example, bidders can be geographically dispersed and bidding can be asynchronous. These conveniences make it easier to obtain a relatively large group of bidders for an object. These, and other special features of online auctions, make it important to re-examine the implications of some key assumptions in auction theory and experiments. In this study, we focus on the assumption that bidders know the distribution of other bidder valuations.

To select the right auction mechanism for environments such as the Internet, one needs to answer two fundamental questions: how the absence of the knowledge of the distribution of bidder valuations affects bidder and auctioneer behavior, and how this change in behavior affects the performance of various auction mechanisms. To address these questions, we conduct laboratory experiments comparing treatments with an unknown distribution of bidder valuations to those with a known distribution of bidder valuations.

The uncertainty about the probability distribution (of bidder valuations, for example) created by missing information is ambiguity. Not knowing important information can affect decision making, as illustrated by the Ellsberg (1961) paradox. Ellsberg's two-color problem uses two urns, one containing 50 red and 50 black balls called the known urn (or the risky urn), and one containing 100 balls in an unknown combination of red and black called the unknown urn (or the ambiguous urn). These two urns represent two distinct types of uncertainty. The first type of uncertainty, present in both urns, is uncertainty as to which outcome will occur: red or black, and is termed risk. The second type of uncertainty, present only in the unknown urn, is uncertainty about the probability of each outcome itself and is termed ambiguity. In Ellsberg experiments, many people bet on red from the known (vs. unknown) urn and on black from the known urn. However, they are indifferent between the two colors when betting on only one urn. This pattern of behavior is inconsistent with any model which uses probabilities, and is called ambiguity aversion. The opposite of ambiguity aversion is called ambiguity loving.

Apart from online auctions, ambiguity is prevalent in many other real-world situations, for example, the success rate of some new drugs or clinical treatments (e.g., Curley, Young and Yates (1989)), the insurance of certain classes of highly ambiguous risks, such as environmental hazards (e.g., Priest (1987)) and terrorist attacks, the usefulness of new features of consumer products (Kahn and Meyer, 1991), the outcomes of $\mathrm{R} \& \mathrm{D}$, incomplete contracting due to unforeseen contingencies, the audit selection procedures of the IRS (Andreoni, Erard and Feinstein (1998)), and initial public offerings (IPOs) of small privately-held firms.

Many researchers have studied ambiguity empirically. In a survey article of empirical and theoretical research on ambiguity, Camerer and Weber (1992) summarize the empirical research into three categories. The first kind of empirical ambiguity research is Ellsberg's original thought experiment and replications of it. The second kind determines the psychological causes of ambiguity. The third kind studies ambiguity in applied settings. While many studies of the first kind find various degrees of ambiguity aversion, Curley and Yates (1989), and Hogarth and Einhorn (1990), among others, find ambiguity loving when subjects face an unknown urn, and a known urn with a low probability of winning. Two studies of ambiguity in experimental markets find mixed results. Camerer and Kunreuther's (1989) study of ambiguity in an insurance market finds that ambiguity about the probability of loss has no systematic effect on insurance prices. Sarin and Weber's (1993) study of ambiguity in an experimental asset market uses a double oral auction and a multi-

\footnotetext{
${ }^{1}$ For surveys of the theoretical literature see McAfee and McMillan (1987) and Klemperer (1999). For a survey of the experimental literature, see Kagel (1995).
} 
unit Vickrey auction. This study finds that the market price for the unambiguous bet is considerably larger than the market price of the ambiguous bet. ${ }^{2}$ Salo and Weber's (1994) study of ambiguity in first price sealed bid auctions finds that ambiguity has no significant effects on bidding behavior. ${ }^{3}$ Moreover, in summarizing studies of ambiguity in applied settings, Camerer and Weber (1992) observe, "These medical and health studies are a little discouraging, because they show less ambiguity aversion, $\cdots$, than is observed or assumed in laboratory experiments (and in theory)." Such mixed results pose the question of whether ambiguity aversion is context dependent. In this paper, we investigate how agents react to ambiguity in one important class of settings, namely first price and second price sealed bid auctions.

There are several different approaches to formally model ambiguity. Among them, maxmin expected utility $^{4}$ (MMEU) and Choquet expected utility ${ }^{5}$ (CEU) models are the most prominent in applications. In this paper we use the $\alpha$-MEU model which is a natural and tractable generalization of the MMEU model. The $\alpha$-MEU, as we discuss in Section 2, allows for both ambiguity averse and ambiguity loving behavior.

Our experiment serves three purposes. First, we extend the large amount of research on auctions to a more realistic setting with the presence of ambiguity, to study how ambiguity affects behavior and to reassess the ranking of first and second price sealed bid auctions in this setting. Second, we study how subjects as auctioneers affect bidder behavior, auctioneer revenue, bidder earnings and auction efficiency. Third, we extend ambiguity research to an important applied setting, to address the question of whether ambiguity aversion is context dependent.

The paper is organized as follows. Section 2 introduces a theoretical model of sealed bid auctions with risk and ambiguity. Section 3 presents the experimental design. Section 4 presents the main results. Section 5 concludes the paper.

\section{Formal Theoretical Development}

This section develops a theoretical auction model incorporating risk and ambiguity. This model guides our experimental design and provides a benchmark for the data analysis.

Three theoretical studies address the role of ambiguity in auctions. Salo and Weber (1995) analyze the first price sealed bid auction using the Choquet expected utility model with a convex capacity. In particular, they consider the case where bidders have a constant relative risk aversion (CRRA) utility function and the Choquet capacity has a power representation. In this case, they show that the equilibrium bidding function is linear. In another study, Lo (1998) analyzes sealed bid auctions using the MMEU framework. Specifically, he derives the equilibrium bidding function for linear utility functions, and compares the first and second price auctions. Using the MMEU framework, Ozdenoren (2002) extends and generalizes the results in Lo. He derives conditions under which risk neutral bidders increase their bids in the first price auction as they become more ambiguity averse. He then uses this result to compare the first and second price auctions.

Our model differs from the above models in two important ways. First we use the $\alpha$-MEU framework to allow for both ambiguity averse and ambiguity loving behavior. This framework is a generalization of both the maxmin and maxmax expected utility models. Second, we consider bidders with general concave utility functions. As a result, previous theory cannot be directly applied to our framework.

Throughout this section, we assume that there are two bidders $i=1,2$. In addition, we assume that there is one indivisible good for sale. In this model, we look at first and second price auctions with independent private values with a reserve price, $r$. Bidders send their bids simultaneously. For simplicity, we assume that

\footnotetext{
${ }^{2}$ In summarizing the different outcomes of the two studies, Camerer and Weber (1992) point out that, in the Sarin and Weber experiments, ambiguity is operationalized as à la Ellsberg.

${ }^{3}$ We discuss the difference between Salo and Weber's design and our design at the end of Section 3.

${ }^{4}$ In the maxmin expected utility model, decision makers have a set of priors and choose an action that maximizes the minimum expected utility over the set of priors.

${ }^{5}$ In the Choquet expected utility model, decision maker's beliefs are represented by a nonadditive probability measure (capacity).
} 
the set of possible valuations of the bidders is $[0,1]$, with $V_{i}$ denoting bidder $i$ 's valuation. Only the bidder knows his own valuation.

Our main departure from previous theoretical and experimental auction literature is the assumption that bidders do not know the valuation distribution. We look at the case, where bidder valuations are known to be independent draws from either $F^{1}(\cdot)$ or $F^{2}(\cdot)$, with positive and a.e.-continuous densities $f^{1}(\cdot)$ and $f^{2}(\cdot)$, respectively. In our experiment, we assume that $F^{2}$ first order stochastically dominates $F^{1}$. Hence, we call $F^{1}$ the low value distribution and $F^{2}$ the high value distribution. For each bidder, the probability, $\delta$, of the event that his opponent's valuation is drawn from the distribution $F^{1}$ is unknown. We define $\delta$ to be the random variable corresponding to the probability that valuation is drawn from $F^{1}$.

In the standard subjective expected utility (SEU) model, each bidder has a subjective prior about the value of $\delta$. However, if a bidder's information about $\delta$ is too vague to be represented by a single prior, it can be represented by a set of priors. In a seminal paper, Gilboa and Schmeidler (1989) provide an axiomatization of the maxmin expected utility model using a set of priors. Expected utility is a special case of MMEU, where the set of beliefs contains only a single probability measure. In this model, a bidder's prior on the event that his opponent's valuation is drawn from the distribution $F^{1}$ is given by a set of probability measures. The bidder's utility is given by the minimum expected utility over this set of priors. Intuitively, a set of priors reflects both ambiguity in the environment and bidder difficulty in forming a well-defined single prior. The min operator, on the other hand, reflects bidder aversion to such ambiguity. To illustrate how MMEU explains Ellsberg type behavior, suppose a decision maker has a linear utility function and the set of priors is $\{(x, 1-x): 0.4 \leq x \leq 0.6\}$, where $x$ is the probability of drawing a red ball and $1-x$ is the probability of drawing a black ball from the unknown urn. The probability of drawing either color from the known urn is 0.5 . In this case, betting $\$ 1$ on either color from the ambiguous urn will give a maxmin expected utility of 0.4 , whereas betting $\$ 1$ on either color from the known urn will give an expected utility of 0.5 .

In general, decision makers may also have preferences that represent ambiguity loving behavior (Heath and Tversky 1990). Such behavior can be captured using the maxmax expected utility model, where the min operator is replaced by the max operator. We do not want to restrict bidders' ambiguity attitude a priori, therefore, we use the $\alpha$-MEU model that allows for both ambiguity averse and ambiguity loving behavior. The $\alpha$-MEU model, axiomatized by Ghirardato et al. (2002), is a generalization of both the maxmin and maxmax expected utility models. In this model, bidders compute the utility of an act using $\alpha$ times the minimum plus $1-\alpha$ times the maximum expected utility over the set of priors. When $\alpha$ equals 1 , this model reduces to MMEU. When $\alpha$ equals 0, it reduces to maxmax EU. Note that the class of preferences this model represents is more general, since $\alpha$ can take all intermediate values.

Formally, let $\Delta$ be a closed and convex subset of the set of distribution functions over $[0,1]$, representing a bidder's belief about the distribution of $\delta$. Let $\underline{\delta}=\min _{G \in \Delta} \int \delta d G(\delta)$ and $\bar{\delta}=\max _{G \in \Delta} \int \delta d G(\delta)$. Note that the set $\Delta$ is subjective and the set $[\underline{\delta}, \bar{\delta}]$ can in general be a strict subset of $[0,1]$. To see this, consider the case where the set $\Delta$ has a single element, $F$. In this case, $\underline{\delta}=\bar{\delta}=$ expected value of $F$. We assume that $\Delta$ is independent of bidder valuations and is common knowledge to all bidders. When there is no ambiguity, the common knowledge assumption reduces to the standard common knowledge assumption in auction theory.

In a first price auction, the bidder with the higher bid above the reserve price receives the object and pays his bid to the seller. However, if both bids are below the reserve price, the object is not sold. Ties are broken by a random device. A bid can be any number in $[0, \infty)$. A reserve price can be any number in $[0,1]$. The payoff for bidder $i$ is given by

$$
\pi_{i}\left(V_{i}, b_{i}, b_{j}, r\right)= \begin{cases}V_{i}-b_{i} & \text { if } b_{i}>b_{j} \text { and } b_{i} \geq r \\ V_{i}-b_{i} \text { or } 0 & \text { if } b_{i}=b_{j} \geq r \\ 0 & \text { if } b_{i}<b_{j} \text { or } b_{i}<r\end{cases}
$$


The bidding strategy of bidder $i$ is given by $s_{i}:[0,1]^{2} \rightarrow[0, \infty)$, mapping own valuation and reserve price into a bid. We assume that, in equilibrium, bidder $i$ knows both his own valuation, $V_{i}$, and bidder $j$ 's strategy, $s_{j}$, but not $j$ 's valuation. Bidder $i$ best replies to bidder $j$ 's strategy given his valuation, the reserve price and his beliefs $\Delta$.

In our framework, the set of priors, $\Delta$, captures the ambiguity attitude. In order to capture bidders' risk attitude, we use a concave utility function, $u(\cdot)$, with $u(0)=0, u^{\prime}>0$, and $u^{\prime \prime}<0$. Given the other bidder's strategy $s_{j}$, the reserve price $r$, and bidder $i$ 's own valuation $V_{i}$, bidder $i$ chooses his bid by maximizing

$$
U_{i}\left(b_{i} ; V_{i}, r, s_{j}\right) \equiv u\left(V_{i}-b_{i}\right) F_{\alpha}\left[s_{j}^{-1}\left(b_{i}, r\right)\right],
$$

where $s_{j}^{-1}\left(b_{i}, r\right)$ is the partial inverse of $s_{j}$ with respect to its first argument, which, in equilibrium, is bidder $j$ 's value, and $F_{\alpha}=(\alpha \underline{\delta}+(1-\alpha) \bar{\delta}) F^{1}+[1-(\alpha \underline{\delta}+(1-\alpha) \bar{\delta})] F^{2}$ is the bidder's belief about $\delta$. In other words, an $\alpha$-MEU bidder will behave as if he believes that his opponent's valuation is drawn from $F^{1}$ with probability $\alpha \underline{\delta}+(1-\alpha) \bar{\delta}$ and from $F^{2}$ with probability $1-(\alpha \underline{\delta}+(1-\alpha) \bar{\delta})$. The derivation of Eq. (2) is in Appendix A.

Strategies $s_{1}$ and $s_{2}$ are equilibrium strategies if

$$
U_{i}\left(s_{i}\left(V_{i}, r\right) ; V_{i}, r, s_{j}\right) \geq U_{i}\left(b_{i} ; V_{i}, r, s_{j}\right)
$$

for all $\left(V_{i}, r\right) \in[0,1]^{2}, b_{i} \in[0, \infty), i=1,2$, and $j=3-i$. In the following proposition, we characterize the symmetric equilibrium strategy.

Proposition 1 The symmetric equilibrium bidding strategy is characterized by the following:

(a) if $V<r$, then $s(V, r) \in[0, r)$;

(b) if $V=r$, then $s(V, r) \in[0, r]$; and

(c) if $V>r$, then $r<s(V, r)<V$, and $s$ is characterized by

$$
\frac{\partial s}{\partial V}(V, r)=\frac{F_{\alpha}^{\prime}(V)}{F_{\alpha}(V)} \frac{u[V-s(V, r)]}{u^{\prime}[V-s(V, r)]}
$$

Proof: See Appendix A.

This Proposition characterizes the symmetric equilibrium bidding strategies for an $\alpha$-MEU bidder. Eq. (3) in Proposition 1 is analogous to the equilibrium characterization for the no-ambiguity case by Riley and Samuelson (1981) and Milgrom and Weber (1982). We now use a particular specification for $F^{1}$ and $F^{2}$ in order to investigate further properties of the bidding function. We use this specification later in the experiments. In Section 3, we discuss why we choose these functional forms.

We use the following specifications for $F^{1}$ and $F^{2}$. The low value distribution $F^{1}$ corresponds to the case where we first choose the interval $\left[0, \frac{1}{2}\right]$ with probability $\frac{3}{4}$ and the interval $\left(\frac{1}{2}, 1\right]$ with probability $\frac{1}{4}$. Subsequently, we choose the valuation from the chosen interval uniformly. Similarly, the high value distribution $F^{2}$ corresponds to the case where we first choose the interval $\left[0, \frac{1}{2}\right]$ with probability $\frac{1}{4}$ and the interval $\left(\frac{1}{2}, 1\right]$ with probability $\frac{3}{4}$. Again, we then choose the valuation from the chosen interval uniformly. More precisely, the two distribution functions are specified as follows:

$$
\begin{aligned}
& F^{1}(x)=\left\{\begin{array}{ccc}
\frac{3}{2} x & \text { if } & 0 \leq x \leq \frac{1}{2} \\
\frac{3}{4}+\left(x-\frac{1}{2}\right) \frac{1}{2} & \text { if } & \frac{1}{2}<x \leq 1
\end{array}\right. \\
& F^{2}(x)=\left\{\begin{array}{ccc}
\frac{1}{2} x & \text { if } & 0 \leq x \leq \frac{1}{2} \\
\frac{1}{4}+\left(x-\frac{1}{2}\right) \frac{3}{2} & \text { if } & \frac{1}{2}<x \leq 1
\end{array} .\right.
\end{aligned}
$$


[Figure 1 about here.]

Figure 1 presents graphs of the cumulative distribution functions $F^{1}$ and $F^{2}$. Note that neither $F^{1}$ nor $F^{2}$ is uniform. A non-uniform distribution in first price auctions allows separation of equilibrium bidding functions from linear rules of thumb. We elaborate on this issue in Section 4.

Recall that $F_{\alpha}=(\alpha \underline{\delta}+(1-\alpha) \bar{\delta}) F^{1}+[1-(\alpha \underline{\delta}+(1-\alpha) \bar{\delta})] F^{2}$. Thus, $F_{\alpha}$ can be expressed as:

$$
\begin{aligned}
F_{\alpha}(x) & =\left\{\begin{array}{cc}
\theta x & \text { if } 0 \leq x \leq \frac{1}{2} \\
\frac{1}{2} \theta+\left(x-\frac{1}{2}\right)(2-\theta) & \text { if } \frac{1}{2}<x \leq 1
\end{array}\right. \\
& =\left\{\begin{array}{cl}
\theta x & \text { if } \quad 0 \leq x \leq \frac{1}{2} \\
(\theta-1)+(2-\theta) x & \text { if } \quad \frac{1}{2}<x \leq 1,
\end{array}\right.
\end{aligned}
$$

where

$$
\begin{aligned}
\theta & =(\alpha \underline{\delta}+(1-\alpha) \bar{\delta}) \frac{3}{2}+[1-(\alpha \underline{\delta}+(1-\alpha) \bar{\delta})] \frac{1}{2} \\
& =(\alpha \underline{\delta}+(1-\alpha) \bar{\delta})+\frac{1}{2} .
\end{aligned}
$$

Eq. (5) implies that the higher $\alpha$ is, the lower $\theta$ will be. Recall from Eq. (2) that the higher the parameter $\alpha$ is, the more weight the decision maker puts on the min functional. In this sense, higher values of $\alpha$ reflect more ambiguity aversion. ${ }^{6}$ Consequently, lower values of $\theta$ reflects more ambiguity aversion. To summarize, the parameter, $\alpha$, measures a bidder's ambiguity attitude. The interval, $[\underline{\delta}, \bar{\delta}]$, measures the amount of ambiguity in the environment. Fixing the amount of ambiguity in the environment, $[\underline{\delta}, \bar{\delta}]$, the parameter, $\theta$, also measures a bidder's ambiguity attitude. In the analysis, as we cannot separately identify $\alpha, \underline{\delta}$ and $\bar{\delta}$, we will use $\theta$ as a measure of ambiguity.

In order to identify when a bidder is ambiguity averse (or loving), we first need to know when the bidder is ambiguity neutral, which is characterized by the next proposition.

Proposition 2 When the set of priors is the convex hull of two probability measures, $F^{1}$ and $F^{2}$, and when $\alpha=\frac{1}{2}$, the decision maker is an expected utility maximizer with beliefs given by $\frac{1}{2} F^{1}+\frac{1}{2} F^{2}$, and consequently ambiguity neutral.

Proof: See Proposition 3 of Ghirardato, Klibanoff and Marinacci (1998).

In our case, the set of priors is indeed the convex combination of two probability distributions. This proposition gives us a natural benchmark for the case of ambiguity neutrality, which allows us to formally define ambiguity aversion and ambiguity loving.

Definition 1 When $\alpha=\frac{1}{2}$, the decision maker is ambiguity neutral; when $\alpha>\frac{1}{2}$, the decision maker is ambiguity averse; when $\alpha<\frac{1}{2}$, the decision maker is ambiguity loving.

Using the above parameterizations of $F^{1}$ and $F^{2}$, we can extend the characterization of the bidding function provided in Proposition 1.

\footnotetext{
${ }^{6}$ In fact, Siniscalchi (2002) shows that, once the set of priors $\Delta$ (or, equivalently, the interval $[\underline{\delta}, \bar{\delta}]$ ) is fixed, according to the comparative definitions of ambiguity and ambiguity aversion in Epstein (1999) (provided the set $\Delta$ satisfies the appropriate restrictions on the set of unambiguous events) and in Ghirardato and Marinacci (2002), the index $\alpha$ can be interpreted as an ambiguity aversion parameter.
} 
Corollary 1 With the parameterized distribution functions $F^{1}$ and $F^{2}$, the equilibrium bidding strategy is characterized by

$$
\frac{\partial s}{\partial V}(V, r, \theta)= \begin{cases}\frac{g[V-s(V, r)]}{V} & \text { if } r<V \leq \frac{1}{2} \\ g[V-s(V, r)] h(V, \theta) & \text { if } \max \left\{r, \frac{1}{2}\right\}<V \leq 1,\end{cases}
$$

where

$$
g(z) \equiv \frac{u(z)}{u^{\prime}(z)}
$$

and

$$
h(v, \theta) \equiv \frac{2-\theta}{\theta-1+(2-\theta) v} .
$$

Proof: Substituting Eq. (4) into Eq. (3), we obtain the result.

This more detailed characterization allows us to consider the impact of ambiguity on the bidding function. This issue is addressed by the following Proposition:

Proposition 3 If $r<V \leq \frac{1}{2}, s(V, r, \theta)$ is independent of $\theta$. If $\max \left\{r, \frac{1}{2}\right\}<V_{i} \leq 1, s(V, r, \theta)$ is strictly decreasing in $\theta$.

Proof: See Appendix A.

This proposition shows that, in the range where $\max \left\{r, \frac{1}{2}\right\}<V \leq 1$, an increase in ambiguity aversion (a decrease in $\theta$ ) leads to higher bids, while an increase in ambiguity loving (an increase in $\theta$ ) leads to lower bids. The intuition is the following. When a bidder is more ambiguity averse, she is more pessimistic, which implies that she thinks that her opponent's valuation is more likely to be high. Therefore, she bids more.

In contrast, in a second price auction, the bidder who has the highest bid at least as large as the reserve price receives the object and pays the maximum of the second highest bid and the reserve price to the seller. If both bids are below the reserve price, the object is not sold. Ties are broken by a random device. In this auction, bidding one's true valuation is a weakly dominant strategy, even with ambiguity aversion (see, e.g., Lo (1998)). This leads to our next proposition.

Proposition 4 In a second price sealed bid auction, regardless of the bidder risk and ambiguity attitudes, bidding one's true valuation is a weakly dominant strategy when the valuation is greater than the reserve price. When the valuation is less than or equal to the reserve price, any bid below the reserve price is a weakly dominant strategy.

All theoretical results characterized in this section serve as a guidance for our experimental design and data analysis.

\section{Experimental Design}

The experimental design reflects both theoretical and technical considerations. The design addresses the following objectives: to determine the effect of ambiguity on bidder and auctioneer behavior, to reevaluate the performance of two auction mechanisms in the presence of ambiguity, and to search for factors not considered in the theoretical framework which might also affect bidder and auctioneer behavior. 


\subsection{Economic Environments}

To study the effect of ambiguity on bidder and auctioneer behavior, we chose a $2 \times 2 \times 2$ design. In the first four treatments - first price auctions with known and unknown distributions and second price auctions with known and unknown distributions, each session consists of eight bidders randomly re-matched into groups of two each round. In the other four treatments, each session consists of eight bidders and four auctioneers, each of whom is randomly re-matched into a group of three each round, with each group consisting of one auctioneer and two bidders.

[Table 1 about here.]

Table 1 summarizes the relevant features of the experimental sessions, including information conditions, number of subjects per session, auction mechanisms, treatment abbreviations, exchange rates and the total number of subjects in each of the eight treatments. For each treatment, we conducted five independent sessions using networked computers at the Research Center for Group Dynamics Laboratory at the University of Michigan. This design gives us a total of forty independent sessions and four hundred subjects, ${ }^{7}$ recruited from an email list of Michigan undergraduate and graduate students. ${ }^{8}$ The choice of the $2 \times 2 \times 2$ design is based on the following considerations.

1. Known vs. unknown distributions: we use the treatments with known distributions as a baseline to isolate the effects of ambiguity.

2. Eight-subject vs. twelve-subject treatments: In most previous experiments, experimenters act as auctioneers. To check the robustness of the theoretical predictions, we use subjects as auctioneers in the twelve-subject treatments. This feature marks a major departure from previous experiments.

3. First price vs. second price auctions: One of our main goals in the design is to compare the performance of the two different auction mechanisms in the presence of ambiguity.

One crucial decision in the design was how to implement ambiguity. In many psychology experiments designed to test the Ellsberg paradox, subjects were told nothing about the distribution of the unknown urn. We adopted a similar design in a pilot experiment conducted in April 2001, but found no basis to infer what prior (or set of priors) the subjects used. Thus, for analytical tractability, we narrow ambiguity to a single parameter in this experiment. More specifically, bidder valuations are known to be independent draws from either the low value distribution $F^{1}(\cdot)$ or the high value distribution $F^{2}(\cdot)$. We use the $F^{1}$ and $F^{2}$ specifications from Section 2, with two modifications. First, we re-scale the support to the interval $[0,100]$. Second, we discretize the support to the set $\{1,2, \cdots, 100\}$. For each bidder, the probability $\delta$ of the event that his opponent's valuation is drawn from the distribution $F^{1}$ is unknown. Therefore, we generate ambiguity regarding the valuation distribution through $\delta$.

In the experiment, each bidder's valuation in each round is a random draw from the set $\{1,2, \cdots, 100\}$. We choose $\delta_{0}$ to be 0.70 for two reasons. First, we want the compound distribution to be non-uniform, which precludes $\delta_{0}=0.5$. We choose not to use a uniform distribution, since it might be a focal point in the absence of knowledge about the true distribution. Furthermore, with a uniform distribution, one cannot separate equilibrium bidding strategies from linear rules of thumb in first price auctions (Chen and Plott (1998)). Second, since most previous experiments demonstrate ambiguity aversion, we want to put more weight on the low distribution to create an "optimistic" environment, which leaves room for ambiguity averse bidders to learn. This consideration precludes $\delta_{0}<0.5$. In treatments with known distribution, $\delta_{0}=0.70$ implies that $\bar{\delta}=\underline{\delta}=0.7$. It then follows from Eq. (5) that $\theta=\theta_{0}=1.2$.

\footnotetext{
${ }^{7}$ Despite our explicit announcement in the advertisement that subjects could not participate in the auction experiment more than once and our screening before each session, nine subjects participated twice.

${ }^{8}$ Graduate students in Economics were excluded from the list.
} 


\subsection{Experimental Procedure}

At the beginning of each session, subjects randomly drew a PC terminal number. Then, each subject was seated in front of the corresponding terminal, and given printed instructions. After the instructions were read aloud, subjects completed a set of Review Questions, to test their understanding of the instructions. Afterwards, the experimenter checked answers and answered questions. The instruction period varied between fifteen to thirty minutes depending on the treatment. In the eight-subject sessions, all eight subjects were seated in the same room. In the twelve-subject sessions, the four auctioneers went to an adjacent lab after the instruction period while the bidders remained in the original lab. In the treatments with unknown $\delta$, the auctioneers were privately informed of the value of $\delta$ on their screen at the beginning of each round. Each round consisted of the following stages:

1. In each of the twelve-subject treatments, each auctioneer set a reserve price, which could be any integer between 1 and 100, inclusive.

2. Meanwhile, for treatments with an unknown distribution only, each bidder estimated the chance that the valuation of the other bidder in the group was drawn from the high value distribution, i.e., an estimate of $1-\delta$. The bidder also indicated his confidence in his estimate: not confident at all, slightly confident, moderately confident, fairly confident, and very confident. This confidence rating method to elicit ambiguity attitude was proposed and evaluated by psychologists Curley, Young and Yates (1989). Among three different methods to elicit subject ambiguity attitude in decision making, they found this one to be the best.

3. Next, each bidder was informed of the reserve price of his auctioneer (in the twelve-subject treatments) and his own valuation. Note that, in the eight-subject treatments, the reserve price was implicitly set to zero. Then each bidder simultaneously and independently submitted a bid, which could be any integer between 1 and 100, inclusive. Bidders were instructed that if they did not want to buy they could submit any positive integer below the reserve price.

4. Bids were then collected in each group and the object was allocated according to the rules of the auction.

5. Afterwards, each bidder received the following feedback on his screen: his valuation, his bid, the reserve price, the winning bid, whether he received the object, and his payoff.

Each auctioneer received the following feedback: whether the object was sold, his reserve price, the bids in his group, and his payoff.

The subjects did not receive the entire vector of valuations and the corresponding bids, as in some previous studies, to slow down the learning of $\delta$ and thus preserve ambiguity for the initial rounds.

In each treatment, each session lasted thirty rounds with no practice rounds. At the end of thirty rounds, all participants completed a questionnaire to elicit demographic information such as gender, race, age, and the number of siblings, and biological information such as menstrual cycle. The demographic results are reported in a companion paper.

Compared to Salo and Weber's (1994) laboratory study of ambiguity in first price sealed bid auctions, our design has the following characteristics. First, we study both first and second price auctions, while Salo and Weber study first price auctions. Second, we have treatments with and without auctioneers, while Salo and Weber do not have treatments with auctioneers. Third, we use a non-uniform distribution of valuations, while Salo and Weber use the uniform distribution. Fourth, while Salo and Weber also examine unknown number of competitors and dichotomous auctions, we do not. Last, we used four hundred subjects, while 
Salo and Weber used forty-eight subjects. The larger number of observations enables us to obtain more precise estimates in our statistical analysis.

The experiments were conducted from October 2001 to January 2002. Each session lasted from forty minutes to an hour. The exchange rates are presented in Table 1 . The average earning was $\$ 18.78$. Instructions are included in Appendix B. Data are available from the authors upon request.

\section{Results}

We present experimental results in this section. Due to space limitations, survey results are presented in a companion paper. Figure 2 presents the cross plot of bids against values in all eight treatments. The first two columns are for the first price auctions, while the second two columns are for the second price auctions. For each column, the top graph is for the known treatment, while the bottom graph is for the unknown treatment. In all twelve-subject treatments, we exclude observations where valuation is less than or equal to the reserve price. An immediate observation is that in first price auctions most bids are below the value (i.e., below the diagonal), while in second price auctions, bids are often above the values. We now proceed to analyze the difference between treatments with and without ambiguity.

[Figure 2 about here.]

We first estimate bidders' ambiguity attitude in first price auctions by using three different approaches. The nonparametric approach compares bids in the no-ambiguity treatments and those in the ambiguity treatments, and infers bidders' ambiguity attitude based on Proposition 3. This approach imposes minimal assumptions on bidder behavior. The structural approach is based on the equilibrium bidding function to be derived in Corollary 2 and explicitly estimates the ambiguity parameter. Compared to the nonparametric analysis, the structural approach requires more assumptions on bidder utility function. In the third approach, we extend the structural approach by using an individual learning model. While the first two approaches are based on the $\alpha$-MEU model, the third approach is based on the SEU model.

We then examine the effects of ambiguity on bids, reserve prices, revenue, earnings and efficiency. Note that, in all subsequent analysis, we normalize the valuations, reserve prices and bids to be on the interval $[0,1]$, consistent with the notation in our theoretical model.

\subsection{Nonparametric Estimation of Ambiguity Attitude in First Price Auctions}

To estimate bidders' ambiguity attitude, we first compare the bids in the no-ambiguity treatment and those in the ambiguity treatment. As we have a full factorial design, keeping everything else constant, any systematic variations in bids in the ambiguity treatments compared to the no-ambiguity treatments can only be attributed to the variation in the amount of ambiguity. In other words, in both treatments, bidder ambiguity preference, $\alpha$, remains the same, while the interval, $[\underline{\delta}, \bar{\delta}]$, changes.

Recall that both the amount of ambiguity in the environment and bidder's ambiguity attitude are summarized in the parameter $\theta$. Proposition 3 implies that higher $\theta$ leads to lower bids. In the no-ambiguity treatments, $\theta=1.2$ as $\delta=0.7$ is known. Therefore, by comparing bids in the ambiguity treatments and those in the no-ambiguity treatments, we can determine whether $\theta$ in the ambiguity treatments is greater (or less) than 1.2. If bids in the ambiguity treatments are lower, we can infer that $\theta>1.2$, and vice versa. To infer bidder's ambiguity attitudes from $\theta$, we need to assume that the center of the interval, $[\underline{\delta}, \bar{\delta}]$ is at or below 0.7. This assumption puts a weak restriction on the amount of weight on the low value distribution relative to the high value distribution. However, it does not rule out the possibility of putting more than 0.7 weight on the low value distribution, e.g., $[0.4,1.0]$ is centered at 0.7 and thus is allowed by our assumption. A natural place where the interval might be centered is 0.5 , as suggested by the "principle of insufficient 
reason," which Luce and Raiffa (1957, p. 284) attribute to Jacob Bernoulli. This case, too, is covered by this assumption. Under this assumption, if $\theta>1.2$, then $\alpha<1 / 2$, implying ambiguity loving. ${ }^{9}$ If $\theta<1.2$, then bidder ambiguity attitude cannot be determined precisely. ${ }^{10}$ For treatments without $\left(K 1_{8}\right.$ and $\left.U 1_{8}\right)$ and with auctioneers $\left(K 1_{12}\right.$ and $\left.U 1_{12}\right)$ respectively, we compare the mean bids in the no-ambiguity treatment with those in the ambiguity treatment, using the Wilcoxon ranksum test. We also compare the median bids and get similar results.

[Table 2 about here.]

Table 2 reports p-values for the Wilcoxon ranksum tests. The null hypothesis is that mean bids are the same in treatments with and without ambiguity. The alternative hypothesis is that bids are higher in the no-ambiguity treatment. In Round 1, all bids are independent, therefore, we use each individual bid as an independent observation. From Round 2 on, we use a session mean as an independent observation. As we expect the amount of ambiguity to decrease over time, we partition the data into early rounds (Round 1, Rounds 1-3, Rounds 1-5) and later rounds. For each time interval, we compare bids over all values, as well as those in two subranges, $[0,0.5]$ and $(0.5,1]$.

RESULT 1 (Ambiguity Attitude) In first price auctions, bids are lower in the ambiguity treatments compared to the no-ambiguity treatments, implying ambiguity loving.

SUPPORT. The last two columns in Table 2 reports p-values for one-sided Wilcoxon ranksum tests, comparing (mean) bids for treatments with and without ambiguity, for the value range of $(0.5,1]$. For the 8 -subject treatments, it is significant in Round 1. For the 12-subject treatments, it is significant for Rounds 2-30, and weakly significant for Rounds 1-3, 4-30, 6-30 and 1-30.

Result 1 presents a significant finding that bids are lower with the presence of ambiguity. From Proposition 3 and the analysis at the beginning of this subsection, we can infer that bidders are ambiguity loving. This is the first main result of this paper.

Result 1 is surprising, given that a large volume of empirical studies replicating the Ellsberg urn experiment and variations confirm ambiguity aversion. How do we reconcile our result with the "robust" ambiguity aversion finding in psychology? We turn to the literature on the psychological causes of ambiguity aversion.

Note that the interpretation of ambiguity loving in auction settings is not exactly the same as ambiguity loving in individual choice experiments such as the Ellsberg experiment. In our auction setting, ambiguity loving implies that bidders put more weight on the low value distribution when the true underlying weight is unknown. This, in turn, implies that a bidder is pessimistic in thinking that his own valuations are more likely to be low, but optimistic in thinking that his opponent's valuations are also more likely to be low. By contrast, in an Ellsberg urn experiment, ambiguity loving implies a preference for the unknown urn when choosing between known and unknown urns, or pessimism when missing information.

Fox and Tversky (1995) propose the comparative ignorance hypothesis, according to which "ambiguity aversion is driven primarily by a comparison between events or between individuals, and it is greatly reduced or eliminated in the absence of such a comparison." Since our experiment uses a between-subjects design, where subjects participated in a treatment with either known or unknown distributions, not both, this could have contributed to the reduction of ambiguity aversion. In other words, our results are consistent with the comparative ignorance hypothesis. However, this hypothesis does not explain why bidders are ambiguity loving.

\footnotetext{
${ }^{9}$ To see this, note that if $\theta>1.2$, then $\alpha \underline{\delta}+(1-\alpha) \bar{\delta}>0.7$. Under our assumption, $(\underline{\delta}+\bar{\delta}) / 2 \leq 0.7$. So when $\alpha=1 / 2$, $\alpha \underline{\delta}+(1-\alpha) \bar{\delta} \leq 0.7$. Moreover, $\alpha \underline{\delta}+(1-\alpha) \bar{\delta}$ is decreasing in $\alpha$. Together, these facts imply that $\alpha<1 / 2$.

${ }^{10}$ To see this, suppose $[\underline{\delta}, \bar{\delta}]=[0.3,0.5]$, and suppose $\alpha=0$, which is ambiguity loving, then $\theta=1<1.2$, such a bidder would increase his bid in the ambiguity treatment, even though he is ambiguity loving.
} 
Curley, Yates and Abrams (1986) investigate the plausibility of six hypotheses regarding the psychological sources of ambiguity aversion in a series of urn experiments. Of the six hypotheses, the other-evaluation hypothesis and the hostile nature hypothesis are most relevant for our experiment. The other-evaluation hypothesis states that a decision maker, in making a choice, anticipates that others will evaluate his decision, and therefore, makes the choice that is perceived to be most justifiable to others. The hostile nature hypothesis conjectures that subjects perceive that the process by which the outcomes are determined for the ambiguous option is antagonistic, or at least competitive, towards themselves. Comparing our experiment to previous individual choice experiments, we note that ambiguity is particularly salient in the Ellsberg urn experiments, where a decision maker's only influence on the outcome is the choice of the urn. However, in the auction context, ambiguity is not as salient. If we extend the other-evaluation and hostile nature hypotheses to auctions, the outcome to be evaluated is affected by the underlying distribution, as well as by bidder and auctioneer strategies. In this complex environment, the prior most justifiable to others could well be such that the experimenter puts more weight on the low value distribution, implying a more competitive outcome-generating process.

Comparing our result to results from Ellsberg urn and market experiments, we conclude that decision makers' ambiguity attitudes are context dependent. It also supports Camerer and Weber's (1992) summary of medical and health studies which show less ambiguity aversion "than is observed or assumed in laboratory experiments (and in theory)."

In order to get an idea of the magnitude of the ambiguity parameter and the dynamics of ambiguity attitude, we proceed to estimate the ambiguity parameter using a structural approach in the $\alpha$-MEU framework and an individual learning model in the SEU framework.

\subsection{Structural Estimation of Ambiguity Parameter in First Price Auctions}

In the previous subsection, we determined that bidders are ambiguity loving from comparison of bids in the two treatments. To get an idea of the magnitude of the ambiguity parameter, $\theta$, we now use the structural approach, to directly estimate $\theta$. As is common in the structural approach, we need additional assumptions to make the model tractable. Our first assumption is that an ambiguity neutral bidder will use the uniform prior in the ambiguity treatment, i.e., $\underline{\delta}+\bar{\delta}=1$. As a result, Eq. (5) implies that $\theta<1$ corresponds to ambiguity aversion, $\theta=1$ corresponds to ambiguity neutrality, and $\theta>1$ corresponds to ambiguity loving. Our second assumption is that bidders have constant relative risk averse (CRRA) utility functions of the form $u(x)=x^{\beta}$, where $\beta>0$. While there has been no consensus on the right model for bidder behavior in first price auctions (see Kagel (1995) and Cox (forthcoming) for surveys of this research), we choose to use CRRA due to its analytical tractability. Because of these assumptions, results on the magnitude of $\theta$ should be taken with caution.

We now compute the equilibrium bidding strategies for an $\alpha$-MEU bidder with a CRRA utility function, using Proposition 1.

Corollary 2 With the parameterized distribution functions $F^{1}$ and $F^{2}$, the equilibrium bidding strategy for a bidder with a CRRA utility function is characterized by:

$s(V, r)= \begin{cases}\frac{V}{1+\beta}+\frac{\beta}{1+\beta} r^{\frac{1+\beta}{\beta}} V^{-\frac{1}{\beta}} & \text { if } r<V \leq \frac{1}{2} \\ \frac{V}{1+\beta}+\frac{\beta}{1+\beta} \frac{\theta-1}{\theta-2}+\frac{\beta}{1+\beta}\left[r^{\frac{1+\beta}{\beta}} 2^{\frac{1}{\beta}}+\frac{\theta-1}{2-\theta}\right]\left(\frac{\theta}{2}\right)^{\frac{1}{\beta}}[\theta-1+(2-\theta) V]^{-\frac{1}{\beta}} & \text { if } r<\frac{1}{2}<V \leq 1 \\ \frac{V}{1+\beta}+\frac{\beta}{1+\beta} \frac{\theta-1}{\theta-2}+\frac{\beta}{(2-\theta)(1+\beta)}[\theta-1+(2-\theta) r]^{\frac{1+\beta}{\beta}}[\theta-1+(2-\theta) V]^{-\frac{1}{\beta}} & \text { if } \frac{1}{2} \leq r<V \leq 1 .\end{cases}$

Proof: See Appendix A. 
We use Corollary 2 to estimate the risk parameter, $\beta$, and ambiguity parameter, $\theta$. In the two control treatments with a known distribution $\left(K 1_{8}\right.$ and $\left.K 1_{12}\right)$, ambiguity does not play a role, as bidders know the value of $\delta$. While treatment $K 1_{8}$ most closely approximates previous experimental studies of first price sealed bid auctions, treatment $K 1_{12}$ serves as a robustness check of whether previous experimental results are sensitive to auctioneers. We use these two treatments to estimate bidder risk attitudes.

We make the simplifying assumption that, within the same treatment, the risk parameter is common and known across individuals. Allowing heterogeneous risk parameters across individuals would clearly fit the data better. However, one has to resort to the computational approach, which requires making ad hoc assumptions about the distribution of risk parameters in the population as well as about independence across individuals and rounds within the same session. Since our main goal is to separate the effects of risk from ambiguity, we assume symmetric bidders to get closed form solutions without distributional assumptions. Moreover, we believe that the main conclusions would remain unchanged even with heterogeneity. Thus, we estimate the following econometric model:

$$
b_{i t}=s\left(V_{i t}, r_{i t} ; \beta, \theta_{0}\right)+\xi_{i t},
$$

where $s(\cdot)$ is the bidding function characterized in Corollary $2 ; b_{i t}$ is the bid submitted by bidder $i$ at round $t ; V_{i t}$ is the private valuation of bidder $i$ at round $t ; r_{i t}$ is the reserve price faced by bidder $i$ at round $t ; \beta$ is the risk parameter; $\theta_{0}=1.2$; and $\xi_{i t}$ is the error term assumed to be orthogonal to both the valuation and the reserve price, i.e., $E\left(\xi_{i t} \mid V_{i t}, r_{i t}\right)=0$. The method of nonlinear least squares is used for parameter estimations. In all estimations, standard errors and confidence intervals are computed by bootstrapping and are adjusted for clustering at the session level, implying that $\xi_{i t}$ is allowed to be heteroscedastic, and correlated across both individuals and rounds, but is independent across sessions. We use the bootstrap procedure to avoid distributional assumptions on $\xi_{i t}$ or relying on asymptotic distribution theory.

[Table 3 about here.]

Table 3 reports the estimates of $\beta$ for treatments $K 1_{8}$ and $K 1_{12}$, respectively. In each estimation, we use only those observations where $V_{i t} \geq r_{i t}$. For each treatment, we first conduct a baseline estimation of $\beta$ with the restriction that $\theta=1.2$. We then repeat the same estimation separately for different subranges of valuations and reserve prices to evaluate the sensitivity of the estimate of $\beta$, since the bidding function has a different functional form for each subrange. Finally, we run a control estimation which jointly estimates $\beta$ and $\theta$. In the control estimation of both treatments, $\theta=1.2$ lies within the $95 \%$ confidence interval, thus justifying the $\theta=1.2$ restriction in the known distribution treatments. The estimated bidder risk parameter is $\beta_{8}=0.3622$ for treatment $K 1_{8}$, and $\beta_{12}=0.5651$ for treatment $K 1_{12}$.

We find that our estimated risk parameters, 0.3622 and 0.5651 , are consistent with recent estimates in private-value auction experiments, such as 0.33 (Cox and Oaxaca (1996)), [0.35, 0.71] (Chen and Plott (1998)) and 0.48 (Goeree, Holt and Palfrey (1999)). However, the estimated risk parameter, $\beta$, is significantly different in treatments with auctioneers. Specifically, bidders seem to be less risk averse in the presence of auctioneers. There could be two reasons for this difference. First, bidders might have perceived the games with and without auctioneers as different games. Indeed, auctioneers and, hence, positive reserve prices cause nearly half the valuations to be below the corresponding reserve prices. ${ }^{11}$ Therefore, it seems that a bidder whose valuation is above the reserve price might take more risk to secure some aspiration level of payoffs. Second, we cannot rule out the possibility that CRRA does not fully capture bidder behavior, and therefore, leading to these two different estimates.

In subsequent analyses, we use the estimated $\beta_{8}=0.3622$ for the eight-subject treatments, and $\beta_{12}=$ 0.5651 for the twelve-subject treatments to isolate the effects of risk and ambiguity. As a robustness check,

\footnotetext{
${ }^{11}$ In treatment $K 1_{12}$, only 637 values out of 1200 observations are above the corresponding reserve prices. We discuss the high reserve prices and its consequences in more detail after Result 5.
} 
we repeat all the subsequent estimation procedures for $\underline{\beta}_{8}=0.32$ and $\bar{\beta}_{8}=0.42$ for the eight-subject treatments, and $\underline{\beta}_{12}=0.40$ and $\bar{\beta}_{12}=0.66$ for the twelve-subject treatments. These alternative values of $\beta$ are reasonable lower and upper bounds based on the estimates of $\beta$ and their respective confidence intervals reported in Table 3.

We now estimate $\theta$ using Corollary 2, with the modification of allowing $\theta$ to vary over time but not over bidders. More specifically, we let $\theta$ be a cubic polynomial of time to partially capture the effects of updating.

[Figure 3 about here.]

Figure 3 presents estimated time paths of $\theta$, together with their bootstrapped confidence intervals, with adjustment for clustering at the session level in treatments with unknown distributions $\left(U 1_{8}\right.$ and $\left.U 1_{12}\right)$. The top row presents the results for the eight-subject treatment $\left(U 1_{8}\right)$, while the bottom row presents the results for the twelve-subject treatment $\left(U 1_{12}\right)$. For each treatment, the first column uses the baseline estimates of the risk parameter $\beta$ from the corresponding treatments with known distributions. The second and third columns serve as robustness checks by using the corresponding lower and upper bounds of $\beta$ respectively. In all six graphs, the estimated ambiguity parameter $\theta$ is at least one, suggesting that bidders are ambiguity loving.

RESULT 2 (Estimation of the Ambiguity Parameter $\theta$ ) : In all rounds, but particularly in the early rounds (1-5), the estimated ambiguity parameter $\theta$ is at least one, with the lower boundaries of all confidence intervals for the eight-subject treatments being at least one, and with the lower boundaries of all confidence intervals for the twelve-subject treatments being approximately one or above one. This rejects ambiguity aversion in both the eight- and twelve-subject treatments. In the eight-subject treatments, starting from round 2, both ambiguity aversion and ambiguity neutrality are rejected in favor of ambiguity loving.

SUPPORT. In all six graphs of Figure 3, we see that the estimated $\theta$ is at least one. Furthermore, the lower boundaries of all confidence intervals for the eight-subject treatments (the top row) are at least one, while the lower boundaries of all confidence intervals for the twelve-subject treatments (the bottom row) are approximately one or above one.

Result 2 confirms Result 1 in establishing ambiguity loving in first price auctions. Apart from the two assumptions discussed earlier, the structural estimation restricts the ambiguity parameter $\theta$ to be the same across individuals in any given round. In the next subsection, we will relax this assumption by modelling individual learning.

\subsection{Learning in First Price Auctions}

We extend the structural approach by explicitly allowing bidders to individually update their priors about the ambiguity parameter $\theta$ based on past observations of their own valuations and the auction outcomes. Unlike mainstream learning literature, which focuses on short, intermediate and long-run learning dynamics, the objective of this analysis is to verify Result 2 by using the entire set of time series data to infer a bidder's prior distribution before the auction. Since there is no consensus on the appropriate updating rule in the $\alpha$-MEU or CEU framework, we use a standard SEU framework with Bayesian updating, a benchmark in learning models. The theoretical derivation of this updating rule is in Appendix A. Here, we outline the theory and the corresponding estimation procedure for our updating rule.

1. We assume that bidders start with some identical prior distribution over the parameter $\delta$, which can be parameterized using a beta distribution. A beta distribution incorporates special cases of interests, such as uniform, unimodal, and bimodal distributions, and has only two parameters, facilitating computation. 
2. In each round, each bidder generates his Bayesian posterior using Bayes rule based on the following signals about either his own valuation or his opponent's valuation.

(a) A bidder observes his own valuation.

(b) In the case where he does not get an object and the object is sold, the bidder is informed of the winning bid in his group and hence infers his opponent's valuation by inverting the symmetric bidding function.

(c) In the case where he does not get an object and the object is not sold, the bidder infers that his opponent's valuation is below the reserve price.

(d) In the case where the bidder gets the object, he infers that his opponent's valuation does not exceed his own valuation.

3. Each bidder's actual posterior is a weighted average of his prior and his generated Bayesian posterior. Note that this approach incorporates Bayesian updating and no updating as special cases. We allow different posterior weights for the first type of signal (based on a bidder's observation of his own valuation) and for the other three types of signals (based on the bidder's observation of auction outcomes), referred to as Weight 1 and Weight 2, respectively.

4. For each parameter combination (two parameters of the beta distribution, Weight 1 and Weight 2), we use the entire time series data set for each bidder to generate predicted bids based on the updating theory outlined above. Then we search for the parameter combination that minimizes the sum of squared deviations ${ }^{12}$ between the actual and generated bids. Weights 1 and 2 are searched on $[0,1]$ with a step size of 0.2. For each combination of Weights 1 and 2, we use an algorithm similar to hill-climbing to locate the minimum of the objective function over the two parameters of the beta distribution. Our computation shows that, conditional on the two weights, the negative of the objective function is single-peaked in the two parameters of the beta distribution.

Recall that in a standard SEU framework, a bidder has a single prior, i.e., in Eq. (5) $\underline{\delta}=\bar{\delta}$. Therefore, in a SEU framework, bidders put less weight on the low value distribution if the estimated mean of $\delta<0.5$, equal weight on the low and high value distributions if the estimated mean of $\delta=0.5$, and more weight on the low value distribution if the estimated mean of $\delta>0.5$.

[Table 4 about here.]

Table 4 presents the results of the updating analysis for the eight-subject as well as the twelve-subject treatments with unknown distributions. In each treatment, we estimate both the baseline and the lower and upper bounds of the risk parameter $\beta$. For each estimation, we present the minimum sum of squared deviations, the two parameters of the initial beta distribution (Par. 1 and Par. 2), the mean of the initial beta distribution implied by the two parameters, and Weights 1 and 2. For each estimation, we also present the percentiles $(2.5,5,95$ and 97.5$)$ of the corresponding bootstrapped ${ }^{13}$ distribution of the implied mean.

RESULT 3 (Prior Inferred from Updating) : The mean of the estimated prior distribution of $\delta$ is 0.8438 in the eight-subject treatment and 0.7500 in the twelve-subject treatment. The hypothesis of subjects putting more weight on the high value distribution is rejected for the twelve-subject treatment, but not for the eightsubject treatment.

\footnotetext{
${ }^{12}$ We use mean squared deviation rather than maximum likelihood because we do not know the distribution of the bid residuals.

${ }^{13}$ In order to reduce the amount of computation, in the bootstrapping procedure, we use a grid of $0,0.5$ and 1 for the Weights 1 and 2. Background computations show that a reduction in the grid increases the minimum sum of squares by, at most, one percent.
} 
SUPPORT. The results in Table 4 indicate that for the eight-subject treatment and the baseline estimate of $\beta=0.3622$, the mean of the estimated prior is 0.8438 , with a two-sided $95 \%$ bootstrapped confidence interval of $[0.1250,0.9688]$. For the twelve-subject treatment and the baseline estimate of $\beta=0.5651$, the mean of the estimated prior is 0.7500 , with a two-sided $95 \%$ bootstrapped confidence interval of [0.5000, 0.8438]. Both point estimates suggest that bidders put more weight on the low value distribution. In addition, in the twelve-subject treatment the one-sided confidence interval indicates that this result is statistically significant at the 5\% level. In the eight-subject treatment, the result is not statistically significant at the 5\% level.

To summarize, we have used three different approaches to determine a bidder's ambiguity attitude. The first approach compares the mean bids in treatments with and without ambiguity and finds that bids are lower in treatments with ambiguity, which implies ambiguity loving. The second approach estimates the ambiguity parameter to be at least one, rejecting ambiguity aversion. Allowing for individual updating, we again infer that the mean of the estimated initial prior distribution of $\delta$ is above 0.5 in both the eight and the twelve-subject treatments. Combining all three approaches, we conclude that ambiguity aversion is rejected in favor of ambiguity loving in first price auctions in our experimental setting.

\subsection{Second Price Auctions}

For second price auctions, we use a structural approach based on Proposition 4, which states that bidding one's true valuation is a weakly dominant strategy with or without ambiguity. To test this hypothesis, we use an OLS regression with clustering at the session level. We use Bid as the dependent variable, and Value as the only independent variable. We do not include a constant because of the theoretical prediction. We conduct the estimation on treatments with known and unknown distributions for both the early (1-5, and 1-10) and later rounds (11-30). We combine both the Known and Unknown treatments in one regression to gain additional efficiency. Results are presented in Table 5.

\section{[Table 5 about here.]}

RESULT 4 (Effects of Ambiguity in Second Price Auctions) : Ambiguity has no significant effect on bids in earlier rounds or later rounds. However, in rounds 1-10 of the Known treatment and rounds 11-30 of both treatments, subjects bid significantly more than their valuations.

SUPPORT. Table 5 presents the OLS regression results for second price auctions. The coefficient estimates show how much subjects bid compared to their valuations. The standard errors are in parentheses. The asterisks next to the standard errors indicate the significance levels in one-sided Wald tests of the null hypothesis of bids being equal to values against the alternative hypothesis of bids exceeding values. The null hypothesis is rejected at the 5\% significance level in rounds 1-10 of the Known treatment and rounds 11-30 of both treatments. The last line of the table displays the Wald $\chi^{2}$ statistics for the equality of coefficients between the known and unknown treatments for the early and later rounds, respectively. None of these statistics is significant at the $10 \%$ significance level.

The finding that ambiguity has no effects on bidding behavior in second price auctions confirms our theoretical prediction. The finding that participants overbid is consistent with previous experimental findings (Kagel, Harstad and Levin (1987)). Interestingly, the extent of overbidding increases in later rounds, which not only confirms that participants do not seem to learn the dominant strategy, but also indicates that they depart further from the dominant strategy in later rounds.

\subsection{Auctioneer Behavior: Reserve Price Setting}

Having examined the effects of ambiguity on bidder behavior in the two auction mechanisms, we now turn to auctioneer behavior. 
We first characterize the optimal reserve price from the auctioneer's perspective. In our experiment, the auctioneer always knows the true distribution of bidder valuations. We assume that the auctioneer also has a CRRA utility function, $u(x)=x^{\lambda}$, where $\lambda>0$. Note that the auctioneer's risk parameter, $\lambda$, could differ from the bidders' risk parameter, $\beta$. In first price auctions, the optimal reserve price depends on the risk attitudes of both the auctioneer $(\lambda)$ and the bidders $(\beta)$, as well as on the ambiguity parameter, $\theta$. Given this set of parameters, we compute the optimal reserve price, presented in Table 7. In second price auctions, we can characterize the auctioneer's optimal reserve price analytically, presented in the following proposition.

Proposition 5 In second price auctions, for any values of $\beta, \lambda \in(0,1]$, the optimal reserve price is given by $\min \left\{\frac{1}{\theta_{0}} \frac{\lambda}{\lambda+1}, 0.5\right\}$, where $\theta_{0}=\delta_{0}+\frac{1}{2}=1.2$.

Proof: See Appendix A.

Since the auctioneer always knows the true distribution of bidder valuations in our experimental setting, Propositions 4 and 5 together imply that the optimal reserve price in the second price auction is the same with or without ambiguity.

For risk averse or risk neutral bidders, we generate the following hypotheses, derived from Propositions 4 and 5 as well as from numerical computations.

HYPOTHESIS 1 In a first price auction, the optimal reserve price should not exceed 0.4167 in treatments without ambiguity. It should not exceed 0.44 in treatments with ambiguity. In a second price auction, the optimal reserve price should not exceed 0.4167 in all treatments.

[Table 6 about here.]

Hypothesis 1 is shown numerically in Table 6 . Table 6 reports the optimal reserve price for first price auctions for each given set of risk parameters $(\beta$ and $\lambda)$ as well as the auctioneer estimate of the bidders' ambiguity parameter, $\theta$. The pattern in Table 6 is consistent with Proposition 5 of Riley and Samuelson (1981), which states that risk-neutral auctioneer's optimal reserve price is a declining function of the degree of bidder risk aversion. The last column of Table 6 reports the optimal reserve price for second price auctions, computed directly from Proposition 5. The computational procedure leading to results in Table 6 is in Appendix A.

HYPOTHESIS 2 In a first price auction, the optimal reserve price is lower (higher) in the case with ambiguity than in the case without, if with ambiguity the seller believes that bidders put less (more) weight on the low value distribution than the actual weight of $\delta_{0}=0.7$, or $\theta<1.2(\theta>1.2)$.

Hypothesis 2 is shown numerically in Table 6. Hypothesis 2 states that, for fixed risk parameters $\beta$ and $\lambda$, the optimal reserve prices increase with $\theta$. This can be seen from the table, since, along each row, the optimal reserve prices increase as $\theta$ increases.

HYPOTHESIS 3 In a second price auction, the optimal reserve price is the same with or without ambiguity.

Hypothesis 3 follows immediately from Proposition 4.

HYPOTHESIS 4 Without ambiguity, the optimal reserve price in a first price auction is less than that in a second price auction.

Hypothesis 4 can be obtained by comparing the two boldfaced columns in Table 6 . 
HYPOTHESIS 5 With ambiguity, the optimal reserve price in a first price auction is less than that in a second price auction, when the auctioneer believes that bidders put less weight on the low value distribution than the actual weight of $\delta=0.7$.

Hypothesis 5 is derived from a combination of Hypotheses 2, 3 and 4.

[Figure 4 about here.]

Figure 4 presents the mean reserve price plus/minus one standard deviation in each of the four treatments. The dashed line is the maximum reserve price predicted by theory in each treatment. Two patterns are immediate. First, in all treatments except $U 1_{12}$, mean reserve prices for individual rounds are often above the maximum predicted by theory. Second, there does not appear to be much learning, as we do not observe any reduction in the standard deviations. We formally test and present the results below.

[Table 7 about here.]

Table 7 reports the average reserve price in early rounds (1-5) and over all rounds (1-30) for each session in each treatment. The last two columns report the alternative hypotheses and the results of the one-tailed permutation tests. In summarizing the results, we use the shorthand $\sim$ to denote a result where the null hypothesis of equality cannot be rejected at the ten percent significance level. We use FPA for first price auctions, and SPA for second price auctions.

\section{RESULT 5 (Reserve Price) :}

1. In ten out of twenty independent sessions, the average reserve price is above the upper bounds of the optimal reserve price.

\section{Effects of information conditions:}

(a) FPA: no ambiguity > ambiguity, significant in early rounds and over all rounds.

(b) SPA: no ambiguity < ambiguity, significant in early rounds; no ambiguity $\sim$ ambiguity over all rounds.

\section{Effects of mechanisms:}

(a) Without ambiguity: FPA $>$ SPA, significant in early rounds; FPA $\sim$ SPA over all rounds.

(b) With ambiguity: FPA $<S P A$, significant in early rounds and over all rounds.

SUPPORT. The last column of Table 7 reports the results of the one-sided permutation tests.

Part 1 of Result 5 shows that in only half of the sessions, the average reserve price is within the limits predicted by Hypothesis 1 . In particular, in three treatments $\left(K 1_{12}, K 2_{12}\right.$ and $\left.U 2_{12}\right)$, the session average reserve prices are too high compared to the optimal reserve price predicted by theory. From Figure 4, the mean reserve price in second price auctions increases over time, consistent with overbidding which increases over time (Table 5). Compared with $K 1_{12}$, the mean reserve price in $U 1_{12}$ is always below the theoretical maximum, consistent with lower bids in first price auctions with ambiguity.

Part 2 (a) is consistent with Hypothesis 2 if the auctioneers believe that bidders weigh the high value distribution more than the actual weight. Part 2 (b) is consistent with Hypothesis 3 except in the early rounds. Interestingly, Part 3 (a) is not consistent with Hypothesis 4, which predicts that, without ambiguity, the optimal reserve price in a second price auction is more than that in a first price auction. Indeed, we find that Hypothesis 4 is reversed in the early rounds, and that the average reserve price between FPA and SPA 
is indistinguishable over all rounds. Finally, the finding that, with ambiguity, second price auctions have a higher reserve price than first price auctions (Part 3 (b)) is consistent with Hypothesis 5.

Both Parts 2 (a) and 3 (b) of Result 5 suggest that auctioneers believe that the bidders put more weight on the high value distribution than the actual weight. However, this finding does not imply that auctioneers believe that bidders are ambiguity averse, since it includes the case of bidders having a uniform prior (or $\theta=1)$.

All these results are individual level results, regarding how ambiguity affects bidder and auctioneer behavior. We now turn to aggregate results, which have important implications for auction design.

\subsection{Revenue, Earnings and Efficiency}

In this subsection, we present aggregate results. Specifically, we examine the effects of the auction mechanisms (first vs. second price auctions), information conditions (ambiguity vs. no ambiguity treatments), and auctioneers (eight- vs. twelve-subject treatments) on auctioneer revenue, bidder earnings and overall auction efficiency.

In most previous auction experiments, the auctioneer's role is either completely ignored (i.e., the reserve price is set to zero), or the experimenter is the auctioneer (e.g., Lucking-Reiley 2000b). In contrast, in our twelve-subject treatments, subjects are auctioneers, thus enabling revenue comparisons across different treatments with endogenous reserve prices. With a zero reserve price, revenue is a direct consequence of bidder behavior, i.e., the higher the bids, the higher the revenue. However, this relationship is not necessarily true with auctioneers present, since revenue is affected by both bidding behavior and reserve prices.

RESULT 6 (Revenue) : Without ambiguity, FPA generates significantly higher revenue than SPA. With ambiguity and without auctioneers, FPA again generates significantly higher revenue than SPA. With ambiguity and active auctioneers, however, FPA and SPA generates the same amount of revenue.

[Table 8 about here.]

SUPPORT. Table 8 presents the average revenue in the early rounds (1-5) and over all thirty rounds for each session in each treatment. The last two columns report the alternative hypotheses and results of the one-tailed permutation tests for the effects of auction mechanisms and information conditions. The last two rows report the same information for the effects of auctioneers.

Result 6 is consistent with theory. The Revenue Equivalence Theorem states that, without ambiguity and with risk neutrality, FPA and SPA generate the same expected revenue. With risk aversion, bidders bid more in the FPA but not in the SPA; therefore, we obtain the usual result that FPA generates more revenue than the SPA. This results also holds when ambiguity is introduced. When, in addition, active auctioneers are introduced, the revenue dominance of the first price auction disappears.

In addition, we also observe that in FPA in the early rounds of the eight-subject treatment, revenue is significantly less when ambiguity is introduced, a consequence of ambiguity-loving bidders. In SPA, ambiguity does not affect revenue over all rounds of both the eight- and the twelve-subject treatments, which is consistent with theory. Finally, we find that the presence of auctioneers reduces FPA revenue. In first price auctions, the auctioneers would have been significantly better off if they were forced to set a zero reserve price. This is a consequence of auctioneers setting high reserve prices discussed in the previous subsection.

Closely related to auctioneer revenue is bidder earnings. We expect auction mechanisms and information conditions to have opposite effects on bidder earnings compared to auctioneer revenue. We also expect auctioneers to reduce bidder earnings.

[Table 9 about here.] 
Table 9 presents the average bidder earnings in early rounds (1-5) and over all thirty rounds for each session in each treatment. The last two columns report the alternative hypotheses and results of the onetailed permutation tests for the effects of auction mechanisms and information conditions. The last two rows report the same information for the effects of auctioneers. We find that bidder earnings are significantly higher in a second price auction compared to a first price auction. The fact that auctioneers significantly reduce bidder earnings reflects the level of reserve prices.

The last group level result we examine is efficiency. Following the tradition in the auction literature, we define efficiency as equal to one hundred percent if the object goes to the bidder with the higher valuation. We therefore measure the frequency with which the bidder with the higher valuation wins the object. The session level average is reported in Table 10.

RESULT 7 (Efficiency) : Without auctioneers, the average efficiency is $88.83 \%$. With auctioneers, the average efficiency is significantly reduced (to 71.12\%). With ambiguity, FPA is significantly more efficient than SPA.

[Table 10 about here.]

SUPPORT. Table 10 presents the average efficiency for each session in each treatment and the results of the one-sided permutation tests. For each treatment, the difference between the twelve-subject and eight-subject treatments is so obvious that any statistical test is superfluous.

Theoretically, both first and second price auctions should yield one hundred percent efficiency under a zero reserve price. Without auctioneers, we find that average efficiency is fairly close to $90 \%$. This finding is largely consistent with theory and previous experiments. However, in the twelve-subject treatments, efficiency is affected by the reserve prices. For example, with ambiguity, average reserve price in FPA is significantly less than that in SPA, which leads to a higher efficiency in FPA. The fact that the presence of active auctioneers significantly reduces efficiency suggests that the high efficiency estimates of previous experiments might have been an artifact of a zero reserve price.

\section{Conclusions}

In many real world auctions, such as Internet auctions, bidder information regarding other bidders' valuations is vague. To explore the effect of this vagueness on bidder and auctioneer behavior, we study first price and second price sealed bid auctions with independent private values, where the distribution of bidder valuation is not known. We derive the symmetric equilibrium using the $\alpha$-MEU framework. We then test our theoretical predictions to examine how ambiguity affects bidder and auctioneer behavior and to reassess the ranking of the first and second price sealed bid auctions.

Previous experimental studies on ambiguity mostly focus on Ellsberg individual choice experiments, while previous auction experiments mostly assume that the distribution of bidder valuations is common knowledge. Our study extends the experimental auction literature to a more realistic setting with ambiguity. It also extends studies of ambiguity to an important applied setting, to determine whether findings from individual choice experiments are robust in the auction context.

Contrary to the results of many previous studies in Ellsberg urn experiments, in our experimental auction setting, ambiguity aversion is rejected in favor of ambiguity loving. This surprising finding suggests that decision makers' attitudes toward ambiguity are context dependent.

Finally, we extend previous auction experiments by using subjects as auctioneers. We study how auctioneers affect bidder behavior, revenue, earnings and efficiency. Our findings show that auctioneers set reserve prices higher than the theoretical prediction, with interesting consequences for auctioneer revenue, bidder earnings and auction efficiency. Specifically, auctioneers reduce revenue in first price auctions compared 
to treatments without auctioneers. High reserve prices also reduce bidder earnings and auction efficiency. With ambiguity-loving bidders and with real auctioneers, the first price and second price auctions generate the same amount of revenue.

These findings have important implication for auction design in settings with ambiguity (and auctioneers). Our results suggest that from the revenue perspective, the designer ought to be indifferent between first and second price auctions. If efficiency is the most important objective, the designer ought to choose first price auctions.

\section{References}

Andreoni, James, Brian Erard and Jonathan Feinstein. Tax compliance. Journal of Economic Literature 36: 818-860, 1998.

Camerer, Colin and Martin Weber. Recent developments in modelling preferences: uncertainty and ambiguity. Jounrnal of Risk and Uncertainty 5: 325-370, 1992.

Camerer, Colin and Howard Kunreuther. Experimental Markets for Insurance. Journal of Risk and Uncertainty 2, 265-300, 1989.

Casadesus-Masanell, Ramon, Peter Klibanoff and Emre Ozdenoren. Maxmin expected utility over Savage acts with a set of priors. Journal of Economic Theory 92: 33-65, 2000.

Chen, Kay-Yut and Charles Plott. Nonlinear behavior in sealed bid first price auctions. Games and Economic Behavior 25: 34-78, 1998.

Cox, James. First price independent private value auctions. In C. Plott and V. Smith (eds) The Handbook of Experimental Economics Results. Elsevier Press: Amsterdam, forthcoming.

Cox, James, and Ronald Oaxaca. Is bidding behavior consistent with bidding theory for private value auctions, in R. M. Isaac, ed., Research in Experimental Economics 6, Greenwich, Conn.: JAI Press, 131-148, 1996.

Cox, James, Vernon Smith and James Walker. Theory and Individual Behavior of First-Price Auctions. Journal of Risk and Uncertainty 1: 61-99, 1988.

Curley, Shawn and Frank Yates. The center and range of the probability interval as factors affecting ambiguity preferences. Organization Behavior and Human Decision Processes 36: 273-287, 1985.

Curley, Shawn and Frank Yates. An empirical evaluation of descriptive models of ambiguity reactions in choice situations. Journal of Mathematical Psychology 33: 397-427, 1989.

Curley, Shawn, Frank Yates and Richard Abrams. Psychological Sources of Ambiguity Avoidance. Organization Behavior and Human Decision Processes 38, 230-256, 1986.

Curley, Shawn, Mark Young and Frank Yates. Characterizing physician's perceptions of ambiguity. Medical Decision Making 9: 116-124, 1989.

Ellsberg, Daniel. Risk, ambiguity, and the Savage axioms. Quarterly Journal of Economics 75: 643-669, 1961.

Epstein, Larry. A definition of uncertainty aversion. Quarterly Journal of Economics 66: 579-608, 1999. 
Fox, Craig R. and Amos Tversky. Ambiguity Aversion and Comparative Ignorance. Quarterly Journal of Economics 110(3), August 1995, pages 585-603.

Ghirardato, Paolo, Peter Klibanoff, and Massimo Marinacci. Additivity with Multiple Priors. Journal of Mathematical Economics 30: 405-420, 1998.

Ghirardato, Paolo, Fabio Maccheroni and Massimo Marinacci. Ambiguity from the Differential Viewpoint. Caltech Social Science Working Paper 1130. April 2002.

Ghirardato, Paolo and Massimo Marinacci. Ambiguity Made Precise: A Comparative Foundation. Journal of Economic Theory 102: 251-289, February 2002.

Gilboa, Itzhak. Expected utility theory with purely subjective non-additive probabilities. Journal of Mathematical Economics 16: 141-153, 1987.

Gilboa, Itzhak and David Schmeidler. Maxmin expected utility with a non-unique prior. Journal of Mathematical Economics 18: 141-153, 1989.

Goeree, Jacob, Charles Holt and Thomas Palfrey. Quantal response equilibrium and overbidding in privatevalue auctions. Working paper, California Institute of Technology, 1999.

Heath, $\mathrm{C}$ and Amos Tversky. Preference and Belief - Ambiguity and Competence in Choice under Uncertainty. Contemporary Issues in Decision Making: 93-123, 1990.

Hogarth, Robin and Hillel Einhorn. Venture theory: a model of decision weights. Management Science 36: 780-803, 1990.

Kagel, John. Auctions: A Survey of Experimental Research. In J. Kagel and A. Roth (eds) Handbook of Experimental Economics. Princeton University Press: Princeton, New Jersey, 1995.

Kagel, John, Ronald Harstad and Dan Levin. Information Impact and Allocation Rules in Auctions with Affiliated Private Values: A Laboratory Study. Econometrica 55 (6), 1275-1304, November, 1987.

Kahn, Barbara and Robert Meyer. Consumer multiattribute judgments under attribute weight uncertainty. Journal of Consumer Research 17: 508-522, 1991.

Klemperer, Paul. Auction Theory: A Guide to the Literature. Journal of Economic Surveys 13: 227-286. 1999.

Lo, Kin-Chung. Sealed Bid Auctions with Uncertainty Averse Bidders. Economic Theory 12 (1) :1-20, July 1998.

Luce, R. Duncan and Howard Raiffa. Games and Decisions: Introduction and Critical Survey. John Wiley and Sons, New York, 1957.

Lucking-Reiley, David. Auctions on the Internet: What's Being Auctioned, and How? Journal of Industrial Economics 48: 227-252, 2000a.

Lucking-Reiley, David. Field Experiments on the Effects of Reserve Prices in Auctions: More Magic on the Internet. Manuscript. University of Arizona, December $2000 \mathrm{~b}$.

Milgrom, Paul and Robert Weber. A Theory of Auctions and Competitive Bidding. Econometrica 50 (5): 1089-1122, September 1982. 
Ozdenoren, Emre. Auctions and bargaining with a set of priors. University of Michigan working paper. 2002.

Priest, George. The current insurance crisis and modern Tort law. Yale Law Journal 96: 1521-1590, 1987.

Riley, John and William Samuelson. Optimal Auctions. American Economic Review 71 (3): 381-392, June 1981.

Salo, Ahti and Martin Weber. First-Price Sealed-Bid Auctions: An Experiment on Probabilistic Risk. Universität Mannheim Working Paper, 1994.

Salo, Ahti and Martin Weber. Ambiguity Aversion in First-Price Sealed-Bid Auctions. Journal of Risk and Uncertainty 11: 123-137, 1995.

Sarin, Rakesh and Martin Weber. Effects of Ambiguity in Market Experiments. Management Science 39:602-615, 1993.

Savage, L. The Foundation of Statistics. John Wiley and Sons, New York, 1954.

Schmeidler, David. Subjective probability and expected utility without additivity. Econometrica 57: 571$587,1989$.

Siniscalchi, Marciano. Vector-Adjusted Expected Utility. Manuscript, Princeton University, 2002. 


\section{APPENDIX A.}

Derivation of Eq. (2): Conditional on $\delta \in[0,1]$, the distribution of the opponent's valuations is given by $\delta F^{1}+(1-\delta) F^{2}$. Then, in light of the $\alpha$-MEU theory, bidder $i$ 's utility is a weighted average of the utility of a maxmin EU bidder (weight $\alpha$ ) and a maxmax EU bidder (weight $1-\alpha$ ), where the set of beliefs over $\delta$ is given by $\Delta$. Then, conditional on the opponent strategy being $s_{j}$ and using the shorthand notation $\pi_{i}$ for $\pi_{i}\left[V_{i}, b_{i}, s_{j}\left(V_{j}, r\right), r\right]$, the bidder $i$ 's payoff $U\left(b_{i} ; V_{i}, r, s_{j}\right)$ is given by

$$
\begin{aligned}
U_{i}\left(b_{i} ; V_{i}, r, s_{j}\right)= & \alpha \min _{G \in \Delta}\left\{\int_{0}^{1} \int_{0}^{1} u\left(\pi_{i}\right) d\left[\delta F^{1}\left(V_{j}\right)+(1-\delta) F^{2}\left(V_{j}\right)\right] d G(\delta)\right\} \\
& +(1-\alpha) \max _{G \in \Delta}\left\{\int_{0}^{1} \int_{0}^{1} u\left(\pi_{i}\right) d\left[\delta F^{1}\left(V_{j}\right)+(1-\delta) F^{2}\left(V_{j}\right)\right] d G(\delta)\right\} \\
= & \alpha \min _{G \in \Delta}\left\{\left(\int_{0}^{1} \delta d G(\delta)\right)\left[\int_{0}^{1} u\left(\pi_{i}\right) d F^{1}\left(V_{j}\right)\right]+\left(\int_{0}^{1}(1-\delta) d G(\delta)\right)\left[\int_{0}^{1} u\left(\pi_{i}\right) d F^{2}\left(V_{j}\right)\right]\right\} \\
& +(1-\alpha)\left\{\max _{G \in \Delta}\left(\int_{0}^{1} \delta d G(\delta)\right)\left[\int_{0}^{1} u\left(\pi_{i}\right) d F^{1}\left(V_{j}\right)\right]+\left(\int_{0}^{1}(1-\delta) d G(\delta)\right)\left[\int_{0}^{1} u\left(\pi_{i}\right) d F^{2}\left(V_{j}\right)\right]\right\} \\
= & \alpha\left\{\underline{\delta}\left[\int_{0}^{1} u\left(\pi_{i}\right) d F^{1}\left(V_{j}\right)\right]+(1-\underline{\delta})\left[\int_{0}^{1} u\left(\pi_{i}\right) d F^{2}\left(V_{j}\right)\right]\right\} \\
& +(1-\alpha)\left\{\bar{\delta}\left[\int_{0}^{1} u\left(\pi_{i}\right) d F^{1}\left(V_{j}\right)\right]+(1-\bar{\delta})\left[\int_{0}^{1} u\left(\pi_{i}\right) d F^{2}\left(V_{j}\right)\right]\right\} \\
= & \int_{0}^{1} u\left(\pi_{i}\right) d F_{\alpha}\left(V_{j}\right) \\
= & u\left(V_{i}-b_{i}\right) F_{\alpha}\left[s_{j}^{-1}\left(b_{i}, r\right)\right] \chi_{\left\{b_{i} \geq r\right\}},
\end{aligned}
$$

where $F_{\alpha}=(\alpha \underline{\delta}+(1-\alpha) \bar{\delta}) F^{1}+[1-(\alpha \underline{\delta}+(1-\alpha) \bar{\delta})] F^{2}$, and $\chi_{\left\{b_{i} \geq r\right\}}$ is an indicator function.

Proof of Proposition 1: By (2), bidder $i$ solves

$$
s_{i}\left(V_{i}, r\right) \in \underset{b_{i} \in[0, \infty)}{\arg \max } u\left(V_{i}-b_{i}\right) F_{\alpha}\left[s_{j}^{-1}\left(b_{i}, r\right)\right] \chi_{\left\{b_{i} \geq r\right\}} .
$$

If $V_{i}<r$, bidder $i$ can always obtain zero utility by bidding below $r$. On the other hand, bidding at or above $r$ can only generate negative or zero utility. As a result, $s_{i}\left(V_{i}, r\right)<r$ when $V_{i}<r$. If $V_{i}>r$, bidding at or below $r$ or at or above $V_{i}$ leads to zero or negative utility. On the other hand, bidding between $r$ and $V_{i}$ can only generate positive or zero utility. As a result, the range of undominated bids, when $V_{i}>r$, is characterized by $r<s_{i}\left(V_{i}, r\right)<V_{i}$. If $V_{i}=r$, bidding above $r$ can only generate zero or negative utility. As a result, $s_{i}\left(V_{i}, r\right) \leq r$ when $V_{i}<r$. Furthermore, when $V_{i}>r$, the bidding function of bidder $i$ is characterized by the following first order condition:

$$
-u^{\prime}\left[V_{i}-s_{i}\left(V_{i}, r\right)\right] F_{\alpha}\left\{s_{j}^{-1}\left[s_{i}\left(V_{i}, r\right), r\right]\right\}+\frac{u\left[V_{i}-s_{i}\left(V_{i}, r\right)\right] F_{\alpha}^{\prime}\left\{s_{j}^{-1}\left[s_{i}\left(V_{i}, r\right), r\right]\right\}}{\frac{\partial}{\partial V_{j}} s_{j}\left(s_{j}^{-1}\left[s_{i}\left(V_{i}, r\right), r\right], r\right)}=0 .
$$

In a symmetric equilibrium $s_{i}=s_{j}=s$, and hence if follows that if $V>r$,

$$
-u^{\prime}[V-s(V, r)] F_{\alpha}(V)+\frac{u[V-s(V, r)] F_{\alpha}^{\prime}(V)}{\frac{\partial}{\partial V} s(V, r)}=0
$$

which can be rewritten as

$$
\frac{\partial}{\partial V} s(V, r)=\frac{F_{\alpha}^{\prime}(V)}{F_{\alpha}(V)} \frac{u[V-s(V, r)]}{u^{\prime}[V-s(V, r)]}
$$


Proof of Proposition 3: Fix $r$. First, for all $V$ such that $r<V \leq \frac{1}{2}$, Corollary 1 shows that

$$
\frac{\partial s}{\partial V}(V, r, \theta)=\frac{g[V-s(V, r, \theta)]}{V},
$$

and hence the functional form of $s(V, r, \theta)$ is independent of $\theta$.

Now consider all $V$ such that $\underline{v}<V \leq 1$, where $\underline{v} \equiv \max \left\{r, \frac{1}{2}\right\}$. Corollary 1 shows that for this range of values

$$
\frac{\partial s}{\partial V}(V, r, \theta)=g[V-s(V, r)] h(V, \theta) .
$$

Suppose, by contradiction, that there exist $V_{0} \in(\underline{v}, 1]$ and $\theta_{1}, \theta_{2} \in[0.5,1.5], \theta_{1}<\theta_{2}$, such that $s\left(V_{0}, r, \theta_{1}\right)<$ $s\left(V_{0}, r, \theta_{2}\right)$. Define the set $M$ as

$$
M \equiv\left\{V \in\left(\underline{v}, V_{0}\right): s\left(V, r, \theta_{1}\right)=s\left(V, r, \theta_{2}\right)\right\} \cup\{\underline{v}\} .
$$

By continuity of $s(\cdot, r, \theta), M$ is a compact set, and hence $m \equiv \max (M)$ is well-defined. This continuity property also implies that $s\left(V, r, \theta_{1}\right)<s\left(V, r, \theta_{2}\right)$ for all $V \in\left(m, V_{0}\right]$. But because $g(\cdot)$ is strictly increasing, $h(V, \theta)$ is strictly decreasing in $\theta$, and, by construction, $s\left(m, r, \theta_{1}\right)=s\left(m, r, \theta_{2}\right)$, it follows from (7) that

$$
\begin{aligned}
s\left(V_{0}, r, \theta_{1}\right) & =s\left(m, r, \theta_{1}\right)+\int_{m}^{V_{0}} g\left[V-s\left(V, r, \theta_{1}\right)\right] h\left(V, \theta_{1}\right) d V \\
& >s\left(m, r, \theta_{2}\right)+\int_{m}^{V_{0}} g\left[V-s\left(V, r, \theta_{2}\right)\right] h\left(V, \theta_{2}\right) d V \\
& =s\left(V_{0}, r, \theta_{2}\right),
\end{aligned}
$$

which is a contradiction. Therefore it must be the case that $s\left(V, r, \theta_{1}\right) \geq s\left(V, r, \theta_{2}\right)$ for all $V \in(\underline{v}, 1]$ and $\theta_{1}, \theta_{2} \in[0.5,1.5], \theta_{1}<\theta_{2}$.

Now suppose by contradiction that there exists $V_{0} \in(\underline{v}, 1]$ and $\theta_{1}, \theta_{2} \in[0.5,1.5], \theta_{1}<\theta_{2}$, such that $s\left(V_{0}, r, \theta_{1}\right)=s\left(V_{0}, r, \theta_{2}\right)$. Since $h(V, \theta)$ is continuous, positive, and strictly decreasing in $\theta$, there must exist $\varepsilon>0$ and $\gamma>0$ such that

$$
\frac{h\left(V, \theta_{2}\right)}{h\left(V, \theta_{1}\right)}<\frac{1}{1+\varepsilon} \text { for all } V \in\left(V_{0}-\gamma, V_{0}\right) .
$$

In addition, since $s(V, r, \theta)$ is continuous in $V, g(\cdot)$ is continuous, positive, and strictly increasing, $s\left(V, r, \theta_{1}\right) \geq$ $s\left(V, r, \theta_{2}\right)$ for all $V \in\left(\underline{v}, V_{0}\right]$, there must exist $\delta>0$ such that

$$
\frac{g\left[V-s\left(V, r, \theta_{2}\right)\right]}{g\left[V-s\left(V, r, \theta_{1}\right)\right]}<1+\varepsilon \text { for all } V \in\left(V_{0}-\delta, V_{0}\right) .
$$

But then it follows that

$$
g\left[V-s\left(V, r, \theta_{2}\right)\right] h\left(V, \theta_{2}\right)<g\left[V-s\left(V, r, \theta_{1}\right)\right] h\left(V, \theta_{1}\right) \text { for all } V \in\left(V_{0}-\min (\delta, \gamma), V_{0}\right) .
$$

This result, combined with the fact that $s\left[V_{0}-\min (\delta, \gamma), r, \theta_{1}\right] \geq s\left[V_{0}-\min (\delta, \gamma), r, \theta_{2}\right]$, implies that

$$
\begin{aligned}
s\left(V_{0}, r, \theta_{1}\right) & =s\left[V_{0}-\min (\delta, \gamma), r, \theta_{1}\right]+\int_{V_{0}-\min (\delta, \gamma)}^{V_{0}} g\left[V-s\left(V, r, \theta_{1}\right)\right] h\left(V, \theta_{1}\right) d V \\
& >s\left[V_{0}-\min (\delta, \gamma), r, \theta_{2}\right]+\int_{V_{0}-\min (\delta, \gamma)}^{V_{0}} g\left[V-s\left(V, r, \theta_{2}\right)\right] h\left(V, \theta_{2}\right) d V \\
& =s\left(V_{0}, r, \theta_{2}\right),
\end{aligned}
$$


which is a contradiction. Therefore it must be the case that $s\left(V, r, \theta_{1}\right)>s\left(V, r, \theta_{2}\right)$ for all $V \in(\underline{v}, 1]$ and $\theta_{1}, \theta_{2} \in[0.5,1.5], \theta_{1}<\theta_{2}$, meaning that $s\left(V, r, \theta_{1}\right)$ is strictly decreasing in $\theta$ when $V \in(\underline{v}, 1]$.

Proof of Corollary 2: Substituting Eq. (4) into Eq. (3) gives:

$$
\frac{\partial}{\partial V} s(V, r)=\left\{\begin{array}{cll}
\frac{1}{\beta}[V-s(V, r)] \frac{1}{V} & \text { if } \quad r<V \leq \frac{1}{2} \\
\frac{1}{\beta}[V-s(V, r)] \frac{2-\theta}{\theta-1+(2-\theta) V} & \text { if } \quad \max \left\{r, \frac{1}{2}\right\}<V \leq 1
\end{array} .\right.
$$

The solution to this differential equation is:

$$
s(V, r)=\left\{\begin{array}{cll}
c_{1} V^{-\frac{1}{\beta}}+\frac{V}{1+\beta} & \text { if } & r<V \leq \frac{1}{2} \\
\frac{V(\theta-2)+\beta(\theta-1)}{(\theta-2)(1+\beta)}+c_{2}[\theta-1+(2-\theta) V]^{-\frac{1}{\beta}} & \text { if } & \max \left\{r, \frac{1}{2}\right\}<V \leq 1
\end{array},\right.
$$

where $c_{1}$ and $c_{2}$ are determined using the boundary condition $\lim _{V \downarrow r} s(V, r)=r$.

We first consider the case $r<\frac{1}{2}$. In this case

$$
c_{1} r^{-\frac{1}{\beta}}+\frac{r}{1+\beta}=r \Rightarrow c_{1}=\frac{\beta}{1+\beta} r^{\frac{1+\beta}{\beta}} .
$$

Then, by continuity at $V=\frac{1}{2}$,

$$
\frac{\beta}{1+\beta} r^{\frac{1+\beta}{\beta}}\left(\frac{1}{2}\right)^{-\frac{1}{\beta}}+\frac{1}{2(1+\beta)}=\frac{1}{2(1+\beta)}+\frac{\beta}{1+\beta} \frac{\theta-1}{\theta-2}+c_{2}\left(\frac{\theta}{2}\right)^{-\frac{1}{\beta}},
$$

implying

$$
c_{2}=\frac{\beta}{1+\beta}\left[r^{\frac{1+\beta}{\beta}}\left(\frac{1}{2}\right)^{-\frac{1}{\beta}}+\frac{\theta-1}{2-\theta}\right]\left(\frac{\theta}{2}\right)^{\frac{1}{\beta}} .
$$

Next consider the case $r \geq \frac{1}{2}$. In this case, the boundary condition $\lim _{V \downarrow r} s(V, r)=r$. gives:

$$
\frac{r(\theta-2)+\beta(\theta-1)}{(\theta-2)(1+\beta)}+c_{2}(\theta-1+(2-\theta) r)^{-\frac{1}{\beta}}=r,
$$

implying

$$
c_{2}=\frac{\beta}{(2-\theta)(1+\beta)}[\theta-1+(2-\theta) r]^{\frac{1+\beta}{\beta}} .
$$

So we can write the bidding function as follows:

$$
s(V, r)= \begin{cases}\frac{V}{1+\beta}+\frac{\beta}{1+\beta} r^{\frac{1+\beta}{\beta}} V^{-\frac{1}{\beta}} & \text { if } r<V \leq \frac{1}{2} \\ \frac{V}{1+\beta}+\frac{\beta}{1+\beta} \frac{\theta-1}{\theta-2}+\frac{\beta}{1+\beta}\left[r^{\frac{1+\beta}{\beta}} 2^{\frac{1}{\beta}}+\frac{\theta-1}{2-\theta}\right]\left(\frac{\theta}{2}\right)^{\frac{1}{\beta}}[\theta-1+(2-\theta) V]^{-\frac{1}{\beta}} & \text { if } r<\frac{1}{2}<V \leq 1 \\ \frac{V}{1+\beta}+\frac{\beta}{1+\beta} \frac{\theta-1}{\theta-2}+\frac{\beta}{(2-\theta)(1+\beta)}[\theta-1+(2-\theta) r]^{\frac{1+\beta}{\beta}}[\theta-1+(2-\theta) V]^{-\frac{1}{\beta}} & \text { if } \frac{1}{2} \leq r<V \leq 1 .\end{cases}
$$

Proof of Proposition 5: Conditional on $V_{1}, V_{2}$, and $r$, the auctioneer's revenue is given by:

$$
R^{S P A}\left(V_{1}, V_{2}, r\right)=\left\{\begin{array}{cl}
0 & \text { if } \max \left\{V_{1}, V_{2}\right\}<r \\
\max \left\{r, \min \left\{V_{1}, V_{2}\right\}\right\} & \text { if } \max \left\{V_{1}, V_{2}\right\} \geq r
\end{array} .\right.
$$


Let $E U_{A}(r)$ denote the expected utility of the auctioneer when the reserve price is $r$. Then, for all $r \in[0,1]$,

$$
E U_{A}(r)=\int_{0}^{1} \int_{0}^{1} \max \left\{r, \min \left(V_{1}, V_{2}\right)\right\}^{\lambda} \chi_{\left\{\max \left(V_{1}, V_{2}\right) \geq r\right\}} d F\left(V_{2}\right) d F\left(V_{1}\right),
$$

where $\chi_{\{\}}$is an indicator function. By symmetry of the distributions of $V_{1}$ and $V_{2}$, this can be rewritten as:

$$
\begin{aligned}
E U_{A}(r) & =2 \int_{0}^{1} \int_{0}^{1} \max \left\{r, V_{2}\right\}^{\lambda} \chi_{\left\{V_{1} \geq r\right\}} \chi_{\left\{V_{1} \geq V_{2}\right\}} d F\left(V_{2}\right) d F\left(V_{1}\right) \\
& =2 \int_{0}^{1} \int_{0}^{1}\left[r^{\lambda} \chi_{\left\{V_{1} \geq r\right\}} \chi_{\left\{r \geq V_{2}\right\}}+V_{2}^{\lambda} \chi_{\left\{V_{1} \geq V_{2}\right\}} \chi_{\left\{V_{2}>r\right\}}\right] d F\left(V_{2}\right) d F\left(V_{1}\right) \\
& =2 r^{\lambda} F(r)[1-F(r)]+2 \int_{0}^{1} \int_{0}^{1} V_{2}^{\lambda} \chi_{\left\{V_{2}>r\right\}} \chi_{\left\{V_{1} \geq V_{2}\right\}} d F\left(V_{1}\right) d F\left(V_{2}\right) \\
& =2 r^{\lambda} F(r)[1-F(r)]+2 \int_{r}^{1} V_{2}^{\lambda}\left[1-F\left(V_{2}\right)\right] d F\left(V_{2}\right)
\end{aligned}
$$

Recall that the auctioneer always knows the true distribution of the valuations given by $F=\delta_{0} F^{1}+$ $\left(1-\delta_{0}\right) F^{2}$, where $\delta_{0}$ is the true weight placed on $F^{1} . F$ can equivalently be expressed as

$$
F(V)=\left\{\begin{array}{cc}
\theta_{0} V & \text { if } \quad 0 \leq V \leq \frac{1}{2} \\
\left(\theta_{0}-1\right)+\left(2-\theta_{0}\right) V & \text { if } \quad \frac{1}{2}<V \leq 1
\end{array}\right.
$$

where $\theta_{0} \equiv \delta_{0}+1 / 2$. Then, since $\lambda \in(0,1]$, for all $r \in\left(\frac{1}{2}, 1\right)$

$$
\begin{aligned}
\frac{\partial E U_{A}(r)}{\partial r} & =2 \lambda r^{\lambda-1}\left[\left(\theta_{0}-1\right)+\left(2-\theta_{0}\right) r\right]\left(2-\theta_{0}\right)(1-r)+2 r^{\lambda}\left(2-\theta_{0}\right)^{2}(1-r) \\
& -2 r^{\lambda}\left[\left(\theta_{0}-1\right)+\left(2-\theta_{0}\right) r\right]\left(2-\theta_{0}\right)-2 r^{\lambda}\left(2-\theta_{0}\right)^{2}(1-r) \\
& =2 r^{\lambda-1}\left[\left(\theta_{0}-1\right)+\left(2-\theta_{0}\right) r\right]\left(2-\theta_{0}\right)(1-r)\left[\lambda-\frac{r}{1-r}\right] \\
& <0 .
\end{aligned}
$$

Since $E U_{A}(r)$ is continuous at $r=1$, it follows that $E U_{A}(r)<E U_{A}\left(\frac{1}{2}\right)$ for all $r \in\left(\frac{1}{2}, 1\right]$. Therefore, setting $r=1 / 2$ strictly dominates any $r$ above $1 / 2$. For $r<1 / 2$,

$$
\begin{aligned}
\frac{\partial E U_{A}(r)}{\partial r} & =2 \lambda r^{\lambda-1} \theta_{0} r\left(1-\theta_{0} r\right)+2 r^{\lambda} \theta_{0}\left(1-\theta_{0} r\right)-2 r^{\lambda} \theta_{0}^{2} r-2 r^{\lambda} \theta_{0}\left(1-\theta_{0} r\right) \\
& =2 r^{\lambda} \theta_{0}\left[\lambda\left(1-\theta_{0} r\right)-\theta_{0} r\right] \\
& =2 r^{\lambda} \theta_{0}\left[\lambda-(1+\lambda) \theta_{0} r\right] .
\end{aligned}
$$

Because $E U_{A}(r)$ is continuous at $r=1 / 2$, this implies that $E U_{A}(r)$ is single-peaked on $r \in\left[0, \frac{1}{2}\right]$, with the maximum at

$$
r^{*}(\lambda) \equiv \min \left\{\frac{\lambda}{\theta_{0}(1+\lambda)}, \frac{1}{2}\right\} .
$$

Because $r=1 / 2$ strictly dominates any $r$ above $1 / 2$, it follows that the optimum reserve price for an auctioneer with the risk parameter $\lambda$ is $r^{*}(\lambda)$. 


\section{Bayesian Updating:}

In this part, we outline the theoretical basis for our analysis of Bayesian updating. Let $\delta \equiv \operatorname{prob}\left\{V\right.$ is drawn from $\left.F_{1}\right\}$. In the beginning of the auction, each bidder has a single prior belief distribution of $\delta$. We denote this distribution by $G_{0}$ and its density (with respect to the Lebesgue measure) by $g_{0}$. For each $\delta$, the distribution of $V$ can be written as:

$$
F^{\delta}(V)=\delta F^{1}(V)+(1-\delta) F^{2}(V) .
$$

Given this, the overall compounded prior over $V$ is given by

$$
F(V)=\int_{0}^{1} F^{\delta}(V) d G_{0}(\delta) .
$$

Recall that:

$$
\begin{aligned}
F^{1}(V) & =\left\{\begin{array}{ccc}
\frac{3}{2} V & \text { if } & 0 \leq V \leq \frac{1}{2} \\
\frac{1}{2} \frac{3}{2}+\left(V-\frac{1}{2}\right) \frac{1}{2} & \text { if } & \frac{1}{2}<V \leq 1
\end{array}\right. \\
& =\frac{3}{2} V-\max \left\{V-\frac{1}{2}, 0\right\} . \\
F^{2}(V) & =\left\{\begin{array}{ccc}
\frac{1}{2} V & \text { if } & 0 \leq x \leq \frac{1}{2} \\
\frac{1}{2} \frac{1}{2}+\left(V-\frac{1}{2}\right) \frac{3}{2} & \text { if } & \frac{1}{2}<V \leq 1
\end{array}\right. \\
& =\frac{1}{2} V+\max \left\{V-\frac{1}{2}, 0\right\} .
\end{aligned}
$$

Thus,

$$
F^{\delta}(V)=\max \left\{\frac{1}{2} V, \frac{3}{2} V-\frac{1}{2}\right\}+\min \{V, 1-V\} \delta
$$

and the corresponding density is given by

$$
f^{\delta}(V)=\frac{1}{2}+\mathcal{X}_{\left\{V \geq \frac{1}{2}\right\}}+\left[1-2 \mathcal{X}_{\left\{V \geq \frac{1}{2}\right\}}\right] \delta,
$$

where $\mathcal{X}_{\left\{V \geq \frac{1}{2}\right\}}$ is the indicator function of the set $\left\{V \geq \frac{1}{2}\right\}$. Using this, we can compute $F$ as:

$$
\begin{aligned}
F(V) & =\int_{0}^{1} F^{\delta}(V) d G_{0}(\delta) \\
& =\int_{0}^{1}\left[\max \left\{\frac{1}{2} V, \frac{3}{2} V-\frac{1}{2}\right\}+\min \{V, 1-V\} \delta\right] d G_{0}(\delta) \\
& =\max \left\{\frac{1}{2} V, \frac{3}{2} V-\frac{1}{2}\right\}+\min \{V, 1-V\} \mu_{0},
\end{aligned}
$$

where $\mu_{0}$ is the mean of the distribution $G_{0}$.

However, given the availability of signals, each bidder successively updates 59 times during the entire experiment (once each round after seeing his own valuation and once each round except the last after seeing the outcome of that round's auction - see the main text for details). We denote the sequence of these posterior beliefs as $G_{1}, \ldots, G_{59}$. Also, for each $k \in\{1, \ldots, 60\}$ and $t \in\{0, \ldots, 59\}$, let

$$
M_{t}(k) \equiv \int_{0}^{1} \delta^{k} d G_{t}(\delta)
$$

be the $k$-th noncentral moment of $G_{t}$.

The subsequent updating is based on two types of signals. 
First type of signal: In this case, a bidder observes his own valuation, $V=a$. This bidder will then update his belief $G_{t}$ over $\delta$ to $G_{t+1}$. Application of the Bayes rule gives:

$$
g_{t+1}(\delta)=\frac{f^{\delta}(a) g_{t}(\delta)}{\int_{0}^{1} f^{\widehat{\delta}}(a) d G_{t}(\widehat{\delta})}=\frac{\frac{1}{2}+\mathcal{X}_{\left\{a \geq \frac{1}{2}\right\}}+\left(1-2 \mathcal{X}_{\left\{a \geq \frac{1}{2}\right\}}\right) \delta}{\frac{1}{2}+\mathcal{X}_{\left\{a \geq \frac{1}{2}\right\}}+\left(1-2 \mathcal{X}_{\left\{a \geq \frac{1}{2}\right\}}\right) M_{t}(1)} g_{t}(\delta),
$$

Using this formula, it follows that

$$
M_{t+1}(k)=\int_{0}^{1} \delta^{k} g_{1}(\delta) d \delta=\frac{\left(\frac{1}{2}+\mathcal{X}_{\left\{a \geq \frac{1}{2}\right\}}\right) M_{t}(k)+\left(1-2 \mathcal{X}_{\left\{a \geq \frac{1}{2}\right\}}\right) M_{t}(k+1)}{\frac{1}{2}+\mathcal{X}_{\left\{a \geq \frac{1}{2}\right\}}+\left(1-2 \mathcal{X}_{\left\{a \geq \frac{1}{2}\right\}}\right) M_{t}(1)} .
$$

Second type of signal: In this case, a bidder observes that his opponent's valuation $V \leq a$. With the notation analogous to Case 1, we get:

$$
g_{t+1}(\delta)=\frac{F^{\delta}(a) g_{t}(\delta)}{\int_{0}^{1} F^{\widehat{\delta}}(a) d G_{t}(\widehat{\delta})}=\frac{\max \left\{\frac{1}{2} a, \frac{3}{2} a-\frac{1}{2}\right\}+\min \{a, 1-a\} \delta}{\max \left\{\frac{1}{2} a, \frac{3}{2} a-\frac{1}{2}\right\}+\min \{a, 1-a\} M_{t}(1)} g_{t}(\delta) .
$$

Using this formula, it follows that:

$$
M_{t+1}(k)=\int_{0}^{1} \delta^{k} g_{1}(\delta) d \delta=\frac{\max \left\{\frac{1}{2} a, \frac{3}{2} a-\frac{1}{2}\right\} M_{t}(k)+\min \{a, 1-a\} M_{t+1}(k+1)}{\max \left\{\frac{1}{2} a, \frac{3}{2} a-\frac{1}{2}\right\}+\min \{a, 1-a\} M_{t}(1)} .
$$

In each case, in parallel to (8), the overall updated prior over valuations associated with $G_{t}$ is given by:

$$
F_{t}(V)=\left(\frac{1}{2}+\mu_{t}\right) V+\left(1-2 \mu_{t}\right) \max \left\{V-\frac{1}{2}, 0\right\},
$$

where $\mu_{t} \equiv M_{t}(1)$. Consequently, a theoretical bidding function after $t$ rounds of updating can be obtained by replacing $F_{\alpha}$ by $F_{t}$ in Eq. (3), which gives the bidding function in Corollary 2 with $\theta$ replaced by $\mu_{t}+0.5$. Therefore, the sequence $\mu_{1}, \ldots, \mu_{59}$ derived from updating based on the personal experience of a particular bidder is a sufficient statistic for a theoretical prediction of that bidder's sequence of bids. To derive this sequence of first moments, (9) and (10) show that, working backwards, it is necessary to know $M_{59}(1)$, which in turn requires knowing $M_{58}(1)$ and $M_{58}(2)$, which in turn requires knowing $M_{57}(1), M_{57}(2)$ and $M_{57}(3)$, etc., all the way to $M_{0}(1), \ldots, M_{0}(60)$. Therefore, to operationalize this updating procedure, we must specify the first sixty moments of $G_{0}$.

In our application, we parameterize $G_{0}$ by a two-parameter family of beta distributions for which the density $g_{0}$ is given by:

$$
g_{0}(\delta)=\frac{\Gamma(a+b)}{\Gamma(a) \Gamma(b)} \delta^{a-1}(1-\delta)^{b-1},
$$

where the two parameters $a$ and $b$ are positive and $\Gamma$ is the standard Gamma function defined by:

$$
\Gamma(z) \equiv \int_{0}^{\infty} u^{z-1} e^{-u} d u, \quad z>0
$$

and obeying

$$
\Gamma(z+1)=z \Gamma(z), \quad z>0 .
$$

Note that, since $g_{0}$ must integrate to unity, it follows that:

$$
\int_{0}^{1} \delta^{c-1}(1-\delta)^{d-1} d \delta=\frac{\Gamma(c) \Gamma(d)}{\Gamma(c+d)}
$$


for any $c, d>0$. Given the form of $g_{0}$, it follows that, for any $k \in\{1, \ldots, 60\}$,

$$
\begin{aligned}
M_{0}(k) & =\frac{\Gamma(a+b)}{\Gamma(a) \Gamma(b)} \int_{0}^{1} \delta^{a+k-1}(1-\delta)^{b-1} d \delta \\
& =\frac{\Gamma(a+b)}{\Gamma(a) \Gamma(b)} \frac{\Gamma(a+k) \Gamma(b)}{\Gamma(a+b+k)} \\
& =\frac{a(a+1) \ldots(a+k-1)}{(a+b)(a+b+1) \ldots(a+b+k-1)},
\end{aligned}
$$

where the second and third equality use (13) and (12), respectively.

In our estimation, we search for values of $a$ and $b$ common across all bidders that best approximate bidder behavior over all rounds, using the above updating procedure. In addition, we introduce the possibility that bidders do not "fully" update their priors based on observed signals. In particular, we allow bidder posteriors to be weighted averages of their priors and their Bayesian posteriors. To separate the effect in updating based on seeing own valuation from the effect based on seeing the auction outcome, we allow different weights on Bayesian posteriors based on these two types of signals. Letting $w_{1} \in[0,1]$ be the weight on the Bayesian posterior based on seeing one's own valuation and $w_{2} \in[0,1]$ be the weight on the Bayesian posterior based on seeing the auction outcome, (9) is now modified to:

$$
\begin{aligned}
M_{t+1}(k) & =\left(1-w_{i}\right) M_{t}(k)+w_{i} \int_{0}^{1} \delta^{k} g_{1}(\delta) d \delta \\
& =\left(1-w_{i}\right) M_{t}(k)+w_{i} \frac{\left(\frac{1}{2}+\mathcal{X}_{\left\{a \geq \frac{1}{2}\right\}}\right) M_{t}(k)+\left(1-2 \mathcal{X}_{\left\{a \geq \frac{1}{2}\right\}}\right) M_{t}(k+1)}{\frac{1}{2}+\mathcal{X}_{\left\{a \geq \frac{1}{2}\right\}}+\left(1-2 \mathcal{X}_{\left\{a \geq \frac{1}{2}\right\}}\right) M_{t}(1)},
\end{aligned}
$$

where $i \in\{1,2\}$ as necessary, and (10) is now modified to:

$$
\begin{aligned}
M_{t+1}(k) & =\left(1-w_{2}\right) M_{t}(k)+w_{2} \int_{0}^{1} \delta^{k} g_{1}(\delta) d \delta \\
& =\left(1-w_{2}\right) M_{t}(k)+w_{2} \frac{\max \left\{\frac{1}{2} a, \frac{3}{2} a-\frac{1}{2}\right\} M_{t}(k)+\min \{a, 1-a\} M_{t+1}(k+1)}{\max \left\{\frac{1}{2} a, \frac{3}{2} a-\frac{1}{2}\right\}+\min \{a, 1-a\} M_{t}(1)} .
\end{aligned}
$$

These two recursive equations, together with (14) and the theoretical bidding function in Corollary 2 with $\theta$ replaced by $\mu_{t}+0.5$, then serve as a theoretical basis of our updating estimation. It is parameterized by $a$ (Parameter 1), $b$ (Parameter 2), $w_{1}$ (Weight 1), and $w_{2}$ (Weight 2), with the mean of the initial prior given by $M_{0}(1)=a /(a+b)$.

\section{Computation of the Optimal Reserve Price:}

First price auction: Conditional on $V_{1}, V_{2}$, and $r$, the auctioneer's revenue is given by:

$$
R^{F P A}\left(V_{1}, V_{2}, r\right)=\left\{\begin{array}{ll}
0 & \text { if } \max \left\{V_{1}, V_{2}\right\}<r \\
\max \left\{s\left(V_{1}, r\right), s\left(V_{2}, r\right)\right\} & \text { if } \max \left\{V_{1}, V_{2}\right\} \geq r
\end{array},\right.
$$

or equivalently, using the fact that $s(V, r)$ is strictly increasing in $V$,

$$
R^{F P A}\left(V_{1}, V_{2}, r\right)= \begin{cases}0 & \text { if } \max \left\{V_{1}, V_{2}\right\}<r \\ s\left[\max \left\{V_{1}, V_{2}\right\}, r\right] & \text { if } \max \left\{V_{1}, V_{2}\right\} \geq r\end{cases}
$$

Recall that, the auctioneer always knows the true distribution of valuations given by $F=0.7 F^{1}+0.3 F^{2}$, or, equivalently,

$$
F(V)=\left\{\begin{array}{ccc}
1.2 V & \text { if } \quad 0 \leq V \leq \frac{1}{2} \\
\frac{1}{2} 1.2+\left(V-\frac{1}{2}\right) 0.8 & \text { if } \quad \frac{1}{2}<V \leq 1
\end{array} .\right.
$$


Therefore, the distribution of $\max \left\{V_{1}, V_{2}\right\}$ is given by:

$$
G(a)=\left\{\begin{array}{cc}
1.2^{2} a^{2} & \text { if } \quad 0 \leq a \leq \frac{1}{2} \\
(0.2+0.8 a)^{2} & \text { if } \quad \frac{1}{2}<a \leq 1
\end{array}\right.
$$

with the associated density given by:

$$
g(a)=\left\{\begin{array}{cl}
2.88 a & \text { if } \quad 0 \leq a \leq \frac{1}{2} \\
1.6(0.2+0.8 a) & \text { if } \quad \frac{1}{2}<a \leq 1
\end{array} .\right.
$$

Let $E U_{A}(r)$ denote the expected utility of the auctioneer when the reserve price is $r$. Then,

$$
\begin{aligned}
E U_{A}(r) & =\int_{0}^{1} \int_{0}^{1}\left[R^{F P A}\left(V_{1}, V_{2}, r\right)\right]^{\lambda} d F\left(V_{1}\right) d F\left(V_{2}\right) \\
& =\int_{0}^{1} \int_{0}^{1} s\left[\max \left\{V_{1}, V_{2}\right\}, r\right]^{\lambda} \chi_{\left\{\max \left\{V_{1}, V_{2}\right\} \geq r\right\}} d F\left(V_{1}\right) d F\left(V_{2}\right) \\
& =\int_{r}^{1} s(a, r)^{\lambda} g(a) d a .
\end{aligned}
$$

After substituting for $s(a, r)$, using the bidding function in Corollary 2, we search for $r$ that maximizes $E U_{A}(r)$, using the grid $\{0,0.001, \ldots,, 0.999,1\}$ for both the integrand $a$ and the reserve price $r$. The integration is performed by the trapezoid approximation. We repeat this procedure for values of the risk aversion parameters $\beta$ and $\lambda$ on the grid $\{1 / 6,2 / 6, \ldots, 1\}$ and the ambiguity parameter $\theta$ on the grid $\{0.5,0.6, \ldots, 1.5\}$. The results are presented in Table 6 , which shows that the optimal reserve price is strictly increasing in all of $\beta, \lambda$, and $\theta$. Therefore, the highest reserve price under risk aversion or risk neutrality of the bidders and the auctioneers and under ambiguity of the bidders is approximately 0.44 , and it is achieved for $\beta=\lambda=1$ and $\theta=1.5$. In treatments with known distributions, equilibrium bidding is governed by the bidding function in Corollary 2 with $\theta=\theta_{0}=1.2$. Hence the highest reserve price is approximately 0.4167 , which can be shown to be exactly equal to $1 / 2.4$.

Second price auction: In this case the computation is straightforward by using the closed-form solution in Proposition 5, with $\theta_{0}=1.2$. We repeat the computation for values of the risk parameter $\lambda$ on the grid $\{1 / 6,2 / 6, \ldots, 1\}$. The results are presented in the last column of Table 6 . Since $r^{*}(\lambda)$ is strictly increasing in $\lambda$, the highest possible theoretical prediction for the reserve price under risk aversion or risk neutrality of the auctioneers is $1 / 2.4 \cong 0.4167$, regardless of the presence of ambiguity. 
- Each round consists of the following stages:

- Each auctioneer will set a minimum selling price, which can be any integer between 1 and 100, inclusive.

- Meanwhile, each bidder will be asked to give an estimate of the chance that the value of the other bidder in the group is drawn from the high value distribution, i.e., an estimate of $x$. We then ask how confident you are about your estimate. You can choose one among the following five categories: not confident at all, slightly confident, moderately confident, fairly confident, and very confident.

- Bidders are informed of the minimum selling prices of their auctioneers, and then each bidder will simultaneously and independently submit a bid, which can be any integer between 1 and 100, inclusive. If you do not want to buy, you can submit any positive integer below the minimum selling price.

- The bids are collected in each group and the object is allocated according to the rules of the auction explained in the next section.

- Bidders will get the following feedback on their screen: your value, your bid, the minimum selling price, the winning bid, whether you got the object, and your payoff.

Auctioneers will get the following feedback: whether you sold the object, your minimum selling price, the bids, and your payoff.

- The process continues.

\section{Rules of the Auction and Payoffs}

- Bidders: In each round,

- if your bid is less than the minimum selling price, you don't get the object:

Your Payoff $=0$

- if your bid is greater than or equal to the minimum selling price, and:

* if your bid is greater than the other bid, you get the object and pay your bid: Your Payoff = Your Value - Your Bid;

* if your bid is less than the other bid, you don't get the object: Your Payoff $=0$.

* if your bid is equal to the other bid, the computer will break the tie by flipping a fair coin. Therefore,

- with $50 \%$ chance you get the object and pay your bid:

Your Payoff = Your Value - Your Bid;

. with $50 \%$ chance you don't get the object:

Your Payoff $=0$.

- Auctioneers: In each round, you will receive two bids from your group.

- If both bids are less than your minimum selling price, the object is not sold, and :

Your Payoff = 0;

- if at least one bid is greater than or equal to your minimum selling price, you sell the object to the higher bidder and Your Payoff = the Higher Bid.

- For example, if the minimum selling price is 1 , bidder A bids 25 , and bidder B bids 55 , since $55>1$ and $55>25$, bidder B gets the object. Bidder A's payoff = 0; bidder B's payoff $=$ her value -55 ; the auctioneer's payoff $=55$.

- There will be 30 rounds. There will be no practice rounds. From the first round, you will be paid for each decision you make.

- Your total payoff is the sum of your payoffs in all rounds.

- Bidders: the exchange rate is $\$ 1$ for points.

- Auctioneers: the exchange rate is $\$ 1$ for points.

We encourage you to earn as much cash as you can. Are there any questions?

Review Questions: you will have ten minutes to finish the review questions. Please raise your hand if you have any questions or if you finish the review questions. The experimenter will check each participant's answers individually. After ten minutes we will go through the answers together. 
1. Suppose your value is 60 and you bid 62 .

If you get the object, your payoff =

If you don't get the object, your payoff $=$

2. Suppose your value is 60 and you bid 60 .

If you get the object, your payoff $=$

If you don't get the object, your payoff $=$

3. Suppose your value is 60 and you bid 58 .

If you get the object, your payoff =

If you don't get the object, your payoff $=$

4. The minimum selling price is 30 and your bid is 25 , your payoff $=$

5. True or false:

(a) _ If a bidder's value is 25 , it must have been drawn from the low distribution.

(b) _ If a bidder's value is 60, it must have been drawn from the high distribution.

(c) _ You will be playing with the same two participants for the entire experiment.

(d) _ A bidder's payoff depends only on his/her own bid.

(e) _ If you are an auctioneer and your minimum selling price is higher than both bids, your payoff will be zero.

Experiment Instructions - U $\mathbf{2}_{12}$

\section{Rules of the Auction and Payoffs}

- Bidders: In each round,

- if your bid is less than the minimum selling price, you don't get the object:

Your Payoff $=0$

- if your bid is greater than or equal to the minimum selling price, and:

* if your bid is greater than the other bid, you get the object. The price you pay depends on the minimum selling price and the other bid:

- if the other bid is greater than or equal to the minimum selling price, you pay the other bid: Your Payoff = Your Value - the Other Bid;

- if the other bid is less than the minimum selling price, you pay the minimum selling price: Your Payoff = Your Value - the Minimum Selling Price;

* if your bid is less than the other bid, you don't get the object: Your Payoff $=0$.

* if your bid is equal to the other bid, the computer will break the tie by flipping a fair coin. Therefore,

- with $50 \%$ chance you get the object and pay the other bid:

Your Payoff = Your Value - the Other Bid

- with $50 \%$ chance you don't get the object:

Your Payoff $=0$.

- Auctioneers: In each round, you will receive two bids from your group.

- If both bids are less than your minimum selling price, the object is not sold, and :

Your Payoff = 0;

- if both bids are greater than or equal to your minimum selling price, you sell the object to the higher bidder and Your Payoff = the Lower Bid

- if one bid is greater than or equal to your minimum selling price and the other bid is less than your minimum selling price, you sell the object to the higher bidder and Your Payoff = the Minimum Selling Price. 
- For example, if the minimum selling price is 1, bidder A bids 25, and bidder B bids 55, since $55>1$ and $55>25$, bidder $B$ gets the object.

Bidder A's payoff = 0 ;

bidder B's payoff = bidder B's value - bidder A's bid = bidder B's value - 25 ;

the auctioneer's payoff $=25$.

- There will be 30 rounds. There will be no practice rounds. From the first round, you will be paid for each decision you make.

- Your total payoff is the sum of your payoffs in all rounds.

- Bidders: the exchange rate is $\$ 1$ for points.

- Auctioneers: the exchange rate is $\$ 1$ for points.

We encourage you to earn as much cash as you can. Are there any questions?

Review Questions: you will have ten minutes to finish the review questions. Please raise your hand if you have any questions or if you finish the review questions. The experimenter will check each participant's answers individually. After ten minutes we will go through the answers together.

1. Suppose the minimum selling price is 1 , your value is 60 , and you bid 62 .

If the other bid is 59, you get the object. Your payoff $=$

If the other bid is 61 , you get the object. Your payoff $=$ - .

If the other bid is 70, you don't get the object. Your payoff $=$

2. Suppose the minimum selling price is 1 , your value is 60 , and you bid 60 .

If the other bid is 55, you get the object. Your payoff $=\ldots$.

If the other bid is 60 ,

- with _ chance you get the object, your payoff = _;

- with _ chance you don't get the object, your payoff =

If the other bid is 70, you don't get the object. Your payoff $=$

3. Suppose the minimum selling price is 1 , your value is 60 , and you bid 57 .

If the other bid is 55, you get the object. Your payoff $=$

If the other bid is 58, you don't get the object. Your payoff $=$

If the other bid is 70, you don't get the object. Your payoff $=$

4. The minimum selling price is 30 and your bid is 25 , your payoff $=$

5. True or false:

(a) _ If a bidder's value is 25, it must have been drawn from the low distribution.

(b) _ If a bidder's value is 60 , it must have been drawn from the high distribution.

(c) _ You will be playing with the same two participants for the entire experiment.

(d) _ A bidder's payoff depends only on his/her own bid.

(e)__If you are an auctioneer and your minimum selling price is higher than both bids, your payoff will be zero. 


\begin{tabular}{ccccccc}
\hline \hline Information & No. Subjects & Auction & Treatment & \multicolumn{2}{c}{ Exchange Rates } & Total No. \\
\cline { 5 - 6 } Conditions & Per Session & Mechanisms & Abbreviation & Bidders & Auctioneers & Subjects \\
\hline \multirow{3}{*}{ Known } & 8 & 1st Price & $K 1_{8}$ & 20 & - & 40 \\
Distribution & 8 & 2nd Price & $K 2_{8}$ & 20 & - & 40 \\
& 12 & 1st Price & $K 1_{12}$ & 12 & 60 & 60 \\
& 12 & 2nd Price & $K 2_{12}$ & 12 & 60 & 60 \\
\hline \multirow{3}{*}{ Unknown } & 8 & 1st Price & $U 1_{8}$ & 20 & - & 40 \\
Distribution & 12 & 2nd Price & $U 2_{8}$ & 20 & - & 40 \\
& 12 & 1st Price & $U 1_{12}$ & 12 & 60 & 60 \\
\hline \hline
\end{tabular}

Table 1: Features of Experimental Sessions

\begin{tabular}{|c|c|c|c|c|c|c|c|}
\hline \multirow[t]{2}{*}{ Treatment } & \multirow{2}{*}{$\begin{array}{l}\text { Round } \\
1-1\end{array}$} & \multicolumn{2}{|c|}{ All $v$} & \multicolumn{2}{|c|}{$0 \leq v \leq 0.5$} & \multicolumn{2}{|c|}{$0.5<v \leq 1$} \\
\hline & & 0.504 & $(40,40)$ & 0.204 & $(26,19)$ & 0.022 & $(14,21)$ \\
\hline \multirow{6}{*}{ 8-subject } & $2-30$ & 0.133 & $(5,5)$ & 0.133 & $(5,5)$ & 0.183 & $(5,5)$ \\
\hline & $1-3$ & 0.006 & $(5,5)$ & 0.062 & $(5,5)$ & 0.540 & $(5,5)$ \\
\hline & $4-30$ & 0.310 & $(5,5)$ & 0.183 & $(5,5)$ & 0.133 & $(5,5)$ \\
\hline & $1-5$ & 0.012 & $(5,5)$ & 0.038 & $(5,5)$ & 0.310 & $(5,5)$ \\
\hline & $6-30$ & 0.242 & $(5,5)$ & 0.310 & $(5,5)$ & 0.133 & $(5,5)$ \\
\hline & $1-30$ & 0.133 & $(5,5)$ & 0.183 & $(5,5)$ & 0.183 & $(5,5)$ \\
\hline \multirow{7}{*}{ 12-subject } & $1-1$ & 0.555 & $(29,21)$ & 0.89 & $(10,9)$ & 0.680 & $(19,12)$ \\
\hline & $2-30$ & 0.012 & $(5,5)$ & 0.012 & $(5,5)$ & 0.038 & $(5,5)$ \\
\hline & $1-3$ & 0.062 & $(5,5)$ & 0.460 & $(5,5)$ & 0.075 & $(5,5)$ \\
\hline & $4-30$ & 0.012 & $(5,5)$ & 0.012 & $(5,5)$ & 0.093 & $(5,5)$ \\
\hline & $1-5$ & 0.012 & $(5,5)$ & 0.093 & $(5,5)$ & 0.183 & $(5,5)$ \\
\hline & $6-30$ & 0.012 & $(5,5)$ & 0.012 & $(5,5)$ & 0.093 & $(5,5)$ \\
\hline & $1-30$ & 0.012 & $(5,5)$ & 0.012 & $(5,5)$ & 0.062 & $(5,5)$ \\
\hline
\end{tabular}

Table 2: Comparison of Bids with and without Ambiguity

Notes: 1 . The table lists one-sided p-values for the Wilcoxon ranksum tests that bidders bid more under the known distribution than under an unknown distribution of valuations.

2. To assure independence of individual observations, first-period only tests use all the observations individually, while all the other tests use session means. Number of independent observations under the known and unknown distribution is listed in parentheses for each test. 


\begin{tabular}{rrrrrrrr}
\hline \hline Treatment & Restriction on $\theta$ & Sample & Obs. & $\beta$ Coefficient & Std. Error & \multicolumn{2}{c}{ 95\% Confidence Interval } \\
\hline$K 1_{8}$ & $\theta=1.2$ & All Values & 1200 & $\mathbf{0 . 3 6 2 2}$ & $\mathbf{0 . 0 2 4 2}$ & $\mathbf{0 . 3 1 9 9}$ & $\mathbf{0 . 4 1 6 0}$ \\
\hline$K 1_{8}$ & N/A & $V_{i t} \leq 0.5$ & 742 & 0.3573 & 0.0191 & 0.3169 & 0.3900 \\
$K 1_{8}$ & $\theta=1.2$ & $V_{i t}>0.5$ & 458 & 0.3633 & 0.0262 & 0.3185 & 0.4234 \\
\hline$K 1_{8}$ & Unrestricted & All Values & 1200 & 0.3313 & 0.0203 & 0.2863 & 0.3625 \\
& & & & $(\theta=1.288$ & 0.0549 & 1.1809 & $1.3914)$ \\
\hline \hline$K 1_{12}$ & $\theta=1.2$ & $r<V_{i t}$ & 637 & $\mathbf{0 . 5 6 5 1}$ & $\mathbf{0 . 0 4 2 7}$ & $\mathbf{0 . 4 9 5 3}$ & $\mathbf{0 . 6 6 2 1}$ \\
\hline$K 1_{12}$ & $\mathrm{~N} / \mathrm{A}$ & $r<V_{i t} \leq 0.5$ & 192 & 0.4070 & 0.0666 & 0.3190 & 0.5783 \\
$K 1_{12}$ & $\theta=1.2$ & $r \leq 0.5<V_{i t}$ & 384 & 0.5804 & 0.0513 & 0.4971 & 0.6919 \\
$K 1_{12}$ & $\theta=1.2$ & $0.5<r<V_{i t}$ & 61 & 0.4558 & 0.0641 & 0.3928 & 0.6126 \\
\hline$K 1_{12}$ & Unrestricted & $r<V_{i t}$ & 637 & 0.4855 & 0.1021 & 0.3727 & 0.7947 \\
& & & & $(\theta=1.3191$ & 0.1417 & 0.9474 & $1.5051)$ \\
\hline \hline
\end{tabular}

Note: All standard errors and confidence intervals are bootstrapped with adjustment for clustering at session level.

Table 3: Estimation of Bidders' Risk Parameter $(\beta)$ 


\begin{tabular}{|c|c|c|c|c|c|c|c|c|c|c|c|}
\hline Treatments & $\beta$ & MinSS & Par. 1 & $\overline{\text { Par. } 2}$ & Mean & $\overline{\text { Weight } 1}$ & Weight 2 & \multicolumn{4}{|c|}{ Confidence Interval } \\
\hline & & & & & & & & 2.5 & 5 & 95 & 97.5 \\
\hline \multirow{3}{*}{8 -subject } & 0.32 & 10.0273 & 0.0018 & 0.0002 & 0.9062 & 0 & 1 & 0.3438 & 0.5156 & 0.9805 & 0.9922 \\
\hline & 0.3622 & 9.9766 & 0.0016 & 0.0003 & 0.8438 & 0 & 1 & 0.1250 & 0.2031 & 0.9570 & 0.9688 \\
\hline & 0.42 & 9.8495 & 0.5312 & 0.5312 & 0.5000 & 1 & 1 & 0.0703 & 0.0781 & 0.9082 & 0.9219 \\
\hline \multirow{3}{*}{ 12-subject } & 0.40 & 6.9534 & 0.0019 & 0.0001 & 0.9961 & 1 & 1 & 0.8516 & 0.8906 & 0.9980 & 0.9990 \\
\hline & 0.5651 & 6.8936 & 24 & 8 & 0.7500 & 0 & 1 & 0.5000 & 0.5625 & 0.8359 & 0.8438 \\
\hline & 0.66 & 6.9470 & 19.5 & 12.5 & 0.6094 & 0 & 1 & 0.3125 & 0.3906 & 0.7031 & 0.7031 \\
\hline
\end{tabular}

Notes:

1. Par. 1 and Par. 2 refer to the two parameters of the beta distribution, respectively.

2. Weights 1 and 2 are the weights on the Bayesian posteriors based on a bidder's observations of his own valuations, and of auction outcomes, respectively.

Table 4: Estimations of Initial Prior Distribution Using Updating

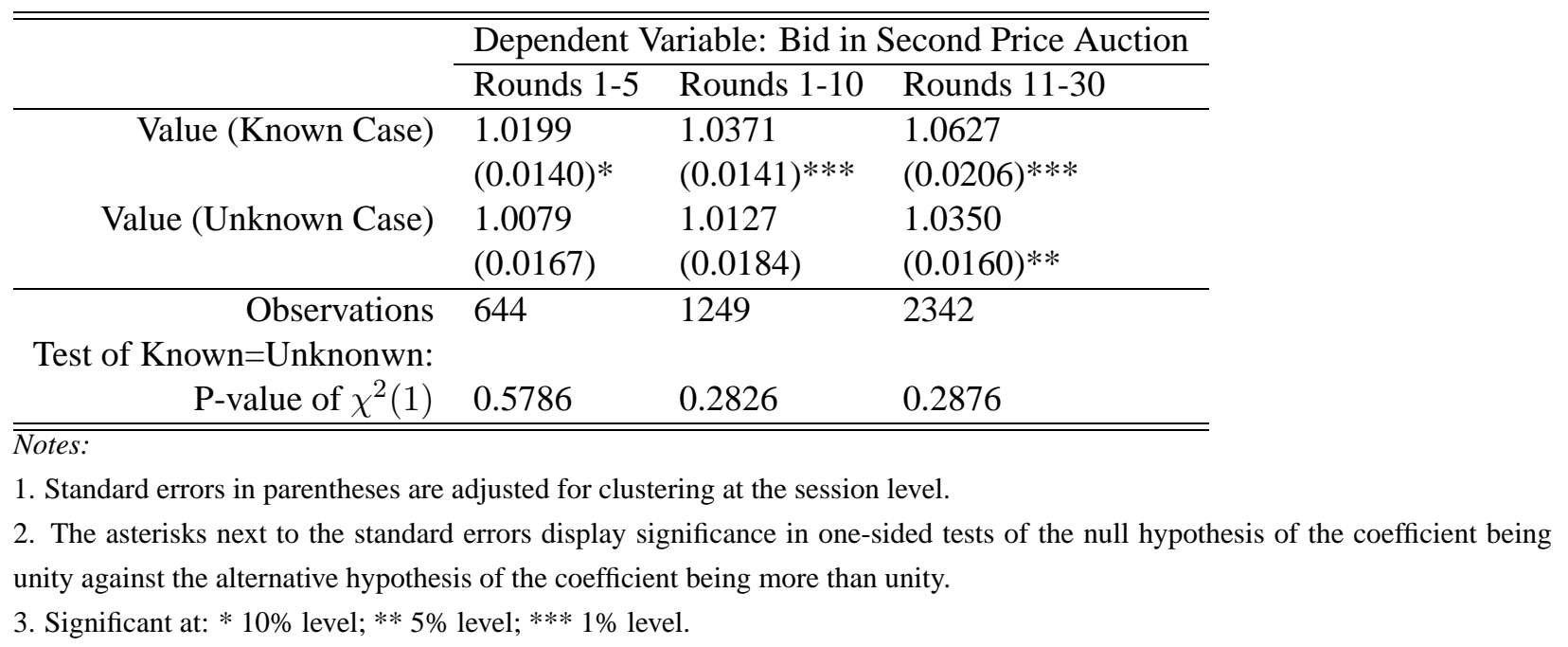

Table 5: Effects of Ambiguity on Bids in Second Price Auctions 


\begin{tabular}{|c|c|c|c|c|c|c|c|c|c|c|c|c|c|}
\hline \multirow[b]{2}{*}{$\beta$} & \multirow[b]{2}{*}{$\lambda$} & \multicolumn{11}{|c|}{ FPA: $\theta$} & \multirow[t]{2}{*}{ SPA } \\
\hline & & 0.5 & 0.6 & 0.7 & 0.8 & 0.9 & 1.0 & 1.1 & 1.2 & 1.3 & 1.4 & 1.5 & \\
\hline \multirow[t]{6}{*}{$1 / 6$} & $1 / 6$ & 0 & 0 & 0 & 0 & 0 & 0 & 0 & 0 & 0 & 0 & 0 & 0.1192 \\
\hline & $2 / 6$ & 0 & 0 & 0 & 0 & 0 & 0 & 0 & 0 & 0 & 0 & 0 & 0.2082 \\
\hline & $3 / 6$ & 0 & 0 & 0 & 0 & 0 & 0 & 0 & 0 & 0 & 0 & 0 & 0.2778 \\
\hline & $4 / 6$ & 0 & 0 & 0 & 0 & 0 & 0 & 0 & 0 & 0 & 0 & 0 & 0.3334 \\
\hline & $5 / 6$ & 0 & 0 & 0 & 0 & 0 & 0 & 0 & 0 & 0 & 0 & 0 & 0.3787 \\
\hline & 1 & 0 & 0 & 0 & 0 & 0 & 0 & 0 & 0 & 0 & 0 & 0 & 0.4167 \\
\hline \multirow[t]{6}{*}{$2 / 6$} & $1 / 6$ & 0 & 0 & 0 & 0 & 0 & 0 & 0 & $\mathbf{0}$ & 0 & 0 & 0 & 0.1192 \\
\hline & $2 / 6$ & 0 & 0 & 0 & 0 & 0 & 0 & 0 & 0 & 0 & 0 & 0 & 0.2082 \\
\hline & $3 / 6$ & 0 & 0 & 0 & 0 & 0 & 0 & 0 & 0 & 0 & 0 & 0 & 0.2778 \\
\hline & $4 / 6$ & 0.0020 & 0.0020 & 0.0020 & 0.0020 & 0.0020 & 0.0020 & 0.0020 & 0.0020 & 0.0020 & 0.0020 & 0.0020 & 0.3334 \\
\hline & $5 / 6$ & 0.0030 & 0.0030 & 0.0030 & 0.0030 & 0.0030 & 0.0030 & 0.0030 & 0.0030 & 0.0030 & 0.0030 & 0.0030 & 0.3787 \\
\hline & 1 & 0.0240 & 0.0240 & 0.0250 & 0.0250 & 0.0260 & 0.0270 & 0.0280 & 0.0290 & 0.0300 & 0.0320 & 0.0340 & 0.4167 \\
\hline \multirow[t]{6}{*}{$3 / 6$} & $1 / 6$ & 0 & 0 & 0 & 0 & 0 & 0 & 0 & 0 & 0 & 0 & 0 & 0.1192 \\
\hline & $2 / 6$ & 0.0020 & 0.0020 & 0.0020 & 0.0020 & 0.0020 & 0.0020 & 0.0020 & 0.0020 & 0.0020 & 0.0020 & 0.0020 & 0.2082 \\
\hline & $3 / 6$ & 0.0200 & 0.0210 & 0.0210 & 0.0220 & 0.0230 & 0.0240 & 0.0250 & 0.0260 & 0.0270 & 0.0290 & 0.0300 & 0.2778 \\
\hline & $4 / 6$ & 0.1010 & 0.1050 & 0.1100 & 0.1140 & 0.1200 & 0.1260 & 0.1320 & 0.1390 & 0.1470 & 0.1550 & 0.1650 & 0.3334 \\
\hline & $5 / 6$ & 0.1710 & 0.1780 & 0.1860 & 0.1940 & 0.2030 & 0.2130 & 0.2230 & 0.2350 & 0.2470 & 0.2600 & 0.2740 & 0.3787 \\
\hline & 1 & 0.2250 & 0.2350 & 0.2460 & 0.2570 & 0.2680 & 0.2810 & 0.2940 & 0.3080 & 0.3220 & 0.3370 & 0.3540 & 0.4167 \\
\hline \multirow[t]{6}{*}{$4 / 6$} & $1 / 6$ & 0.0020 & 0.0020 & 0.0020 & 0.0020 & 0.0020 & 0.0020 & 0.0020 & 0.0020 & 0.0020 & 0.0020 & 0.0020 & 0.1192 \\
\hline & $2 / 6$ & 0.0380 & 0.0390 & 0.0410 & 0.0420 & 0.0440 & 0.0460 & 0.0480 & 0.0500 & 0.0520 & 0.0550 & 0.0580 & 0.2082 \\
\hline & $3 / 6$ & 0.1200 & 0.1250 & 0.1300 & 0.1350 & 0.1410 & 0.1460 & 0.1530 & 0.1590 & 0.1660 & 0.1730 & 0.1810 & 0.2778 \\
\hline & $4 / 6$ & 0.1890 & 0.1970 & 0.2050 & 0.2130 & 0.2220 & 0.2300 & 0.2390 & 0.2480 & 0.2580 & 0.2670 & 0.2780 & 0.3334 \\
\hline & $5 / 6$ & 0.2430 & 0.2540 & 0.2640 & 0.2740 & 0.2850 & 0.2960 & 0.3060 & 0.3170 & 0.3280 & 0.3390 & 0.3500 & 0.3787 \\
\hline & 1 & 0.2870 & 0.3000 & 0.3120 & 0.3240 & 0.3360 & 0.3470 & 0.3590 & 0.3710 & 0.3830 & 0.3940 & 0.4060 & 0.4167 \\
\hline \multirow[t]{6}{*}{$5 / 6$} & $1 / 6$ & 0.0120 & 0.0130 & 0.0130 & 0.0140 & 0.0140 & 0.0150 & 0.0150 & 0.0160 & 0.0160 & 0.0170 & 0.0170 & 0.1192 \\
\hline & $2 / 6$ & 0.0930 & 0.0970 & 0.1000 & 0.1040 & 0.1080 & 0.1120 & 0.1160 & 0.1200 & 0.1250 & 0.1300 & 0.1350 & 0.2082 \\
\hline & $3 / 6$ & 0.1720 & 0.1780 & 0.1850 & 0.1920 & 0.1990 & 0.2060 & 0.2130 & 0.2200 & 0.2270 & 0.2340 & 0.2410 & 0.2778 \\
\hline & $4 / 6$ & 0.2340 & 0.2430 & 0.2520 & 0.2610 & 0.2690 & 0.2780 & 0.2870 & 0.2950 & 0.3040 & 0.3120 & 0.3200 & 0.3334 \\
\hline & $5 / 6$ & 0.2830 & 0.2940 & 0.3050 & 0.3150 & 0.3250 & 0.3350 & 0.3440 & 0.3540 & 0.3630 & 0.3720 & 0.3800 & 0.3787 \\
\hline & 1 & 0.3230 & 0.3360 & 0.3480 & 0.3590 & 0.3700 & 0.3800 & 0.3900 & 0.4000 & 0.4100 & 0.4190 & 0.4280 & 0.4167 \\
\hline \multirow[t]{6}{*}{1} & $1 / 6$ & 0.0370 & 0.0390 & 0.0400 & 0.0410 & 0.0430 & 0.0440 & 0.0460 & 0.0480 & 0.0490 & 0.0510 & 0.0530 & 0.1192 \\
\hline & $2 / 6$ & 0.1290 & 0.1340 & 0.1380 & 0.1430 & 0.1480 & 0.1530 & 0.1580 & 0.1630 & 0.1680 & 0.1730 & 0.1780 & 0.2081 \\
\hline & $3 / 6$ & 0.2040 & 0.2110 & 0.2190 & 0.2260 & 0.2330 & 0.2400 & 0.2470 & 0.2530 & 0.2600 & 0.2670 & 0.2730 & 0.2778 \\
\hline & $4 / 6$ & 0.2620 & 0.2720 & 0.2810 & 0.2900 & 0.2980 & 0.3060 & 0.3140 & 0.3210 & 0.3290 & 0.3360 & 0.3430 & 0.3334 \\
\hline & $5 / 6$ & 0.3090 & 0.3200 & 0.3300 & 0.3400 & 0.3490 & 0.3580 & 0.3670 & 0.3750 & 0.3820 & 0.3900 & 0.3970 & 0.3787 \\
\hline & 1 & 0.3470 & 0.3600 & 0.3710 & 0.3810 & 0.3910 & 0.4000 & 0.4090 & 0.4167 & 0.4250 & 0.4330 & 0.4400 & 0.4167 \\
\hline
\end{tabular}

Table 6: Computed Optimal Reserve Price in First and Second Price Auctions 


\begin{tabular}{rrrrrrll}
\hline \hline Rounds 1-5 & Session 1 & Session 2 & Session 3 & Session 4 & Session 5 & \multicolumn{1}{c}{$H_{1}$} & p-value \\
$K 1_{12}$ & 0.4905 & 0.2285 & 0.4205 & 0.3870 & 0.4135 & $K 1>K 2$ & $0.0278^{* *}$ \\
$U 1_{12}$ & 0.2310 & 0.2075 & 0.3160 & 0.4005 & 0.2500 & $U 1<U 2$ & $0.0476^{* *}$ \\
$K 2_{12}$ & 0.2630 & 0.2700 & 0.3290 & 0.3155 & 0.2150 & $K 1>U 1$ & $0.0516^{*}$ \\
$U 2_{12}$ & 0.4990 & 0.3590 & 0.2360 & 0.3790 & 0.5690 & $K 2<U 2$ & $0.0278^{* *}$ \\
Rounds 1-30 & & & & & & & \\
$K 1_{12}$ & 0.4571 & 0.2938 & 0.4493 & 0.3547 & 0.4341 & $K 1<K 2$ & 0.3611 \\
$U 1_{12}$ & 0.2535 & 0.1707 & 0.3295 & 0.3741 & 0.2522 & $U 1<U 2$ & $0.0000^{* * *}$ \\
$K 2_{12}$ & 0.4964 & 0.4651 & 0.3163 & 0.4763 & 0.2978 & $K 1>U 1$ & $0.0198^{* *}$ \\
$U 2_{12}$ & 0.4448 & 0.5276 & 0.4222 & 0.4152 & 0.5164 & $K 2<U 2$ & 0.1548 \\
\hline \hline
\end{tabular}

Notes:

1. The null hypothesis is that the average reserve price is equal in the two treatments.

2. Significant at: * $10 \%$ level; ** 5\% level; *** $1 \%$ level.

Table 7: Average Reserve Price and Results of Permutation Tests (one-tailed)

\begin{tabular}{rlllllll}
\hline \hline Rounds 1-5 & Session 1 & Session 2 & Session 3 & Session 4 & Session 5 & $H_{1}$ & p-value \\
$K 1_{8}$ & 0.4665 & 0.4685 & 0.4235 & 0.5170 & 0.5485 & $K 1>K 2$ & $0.0040^{* * *}$ \\
$U 1_{8}$ & 0.3705 & 0.4795 & 0.4280 & 0.4420 & 0.3905 & $U 1>U 2$ & $0.0556^{*}$ \\
$K 2_{8}$ & 0.2815 & 0.2665 & 0.2600 & 0.3825 & 0.3795 & $K 1>U 1$ & $0.0397^{* *}$ \\
$U 2_{8}$ & 0.2935 & 0.3870 & 0.4175 & 0.3130 & 0.3990 & $K 2<U 2$ & $0.0992^{*}$ \\
$K 1_{8}$ & 0.4459 & 0.3869 & 0.4443 & 0.4648 & 0.4559 & $K 1>K 2$ & $0.0079^{* * *}$ \\
$U 1_{8}$ & 0.3638 & 0.4419 & 0.4255 & 0.4277 & 0.4499 & $U 1>U 2$ & $0.0159^{* *}$ \\
$K 2_{8}$ & 0.3335 & 0.3265 & 0.3423 & 0.3948 & 0.3506 & $K 1>U 1$ & 0.2341 \\
$U 2_{8}$ & 0.2953 & 0.3653 & 0.3628 & 0.3131 & 0.3588 & $K 2>U 2$ & 0.3730 \\
Rounds 1-30 & & & & & \\
$K 1_{12}$ & 0.4430 & 0.4100 & 0.4625 & 0.3900 & 0.3485 & $K 1>K 2$ & $0.0238^{*}$ \\
$U 1_{12}$ & 0.3540 & 0.4840 & 0.4015 & 0.3085 & 0.3925 & $U 1<U 2$ & 0.2540 \\
$K 2_{12}$ & 0.2760 & 0.3405 & 0.3750 & 0.3925 & 0.3080 & $K 1>U 1$ & 0.2659 \\
$U 2_{12}$ & 0.4120 & 0.4840 & 0.3730 & 0.4550 & 0.3445 & $K 2<U 2$ & $0.0278^{* *}$ \\
Rounds 1-5 & & & & & \\
$K 1_{12}$ & 0.3579 & 0.3918 & 0.3833 & 0.4053 & 0.3523 & $K 1>K 2$ & $0.0317^{* *}$ \\
$U 1_{12}$ & 0.3740 & 0.3968 & 0.3927 & 0.3837 & 0.3844 & $U 1>U 2$ & 0.1190 \\
$K 2_{12}$ & 0.3554 & 0.3405 & 0.3786 & 0.3445 & 0.3434 & $K 1<U 1$ & 0.2063 \\
$U 2_{12}$ & 0.3540 & 0.3821 & 0.4146 & 0.3531 & 0.3455 & $K 2<U 2$ & 0.1111 \\
\hline Rounds 1-30 & & & & \\
$K 1_{8}>K 1_{12}$ & $0.0119 * *$ & $U 1_{8}>U 1_{12}$ & $0.0278 * *$ & $K 2_{8}<K 2_{12}$ & 0.4008 & $U 2_{8}<U 2_{12}$ & $0.0873^{*}$ \\
\hline \hline Notes:
\end{tabular}

1. The null hypothesis is that the average revenue is equal in the two treatments.

2. Significant at: * $10 \%$ level; ** 5\% level; *** $1 \%$ level.

Table 8: Average Revenue and Results of Permutation Tests (one-tailed) 


\begin{tabular}{|c|c|c|c|c|c|c|c|}
\hline Rounds 1-5 & Pession 1 & 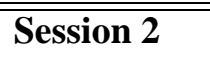 & Session 3 & Session 4 & Session 5 & $H_{1}$ & p-value \\
\hline$K 1_{8}$ & 0.0703 & 0.0653 & 0.0793 & 0.0635 & 0.0175 & $K 1<K 2$ & $0.0000 * * *$ \\
\hline$U 1_{8}$ & 0.0550 & 0.0968 & 0.0555 & 0.0675 & 0.0518 & $U 1<U 2$ & $0.0159 * *$ \\
\hline$K 2_{8}$ & 0.1178 & 0.1530 & 0.1940 & 0.1500 & 0.1393 & $K 1<U 1$ & 0.3333 \\
\hline$U 2_{8}$ & 0.0953 & 0.0690 & 0.0838 & 0.1860 & 0.1448 & $K 2>U 2$ & $0.0952 *$ \\
\hline \multicolumn{8}{|l|}{ Rounds 1-30 } \\
\hline$K 1_{8}$ & 0.0912 & 0.0914 & 0.0883 & 0.0788 & 0.0623 & $K 1<K 2$ & $0.0000 * * *$ \\
\hline$U 1_{8}$ & 0.1194 & 0.0869 & 0.0785 & 0.0912 & 0.0785 & $U 1<U 2$ & $0.0079 * * *$ \\
\hline$K 2_{8}$ & 0.1230 & 0.1426 & 0.1252 & 0.1152 & 0.1299 & $K 1<U 1$ & 0.2421 \\
\hline$U 2_{8}$ & 0.1505 & 0.1115 & 0.1045 & 0.1540 & 0.1366 & $K 2<U 2$ & 0.3532 \\
\hline \multicolumn{8}{|l|}{ Rounds 1-5 } \\
\hline$K 1_{12}$ & 0.0405 & 0.0630 & 0.0723 & 0.0728 & 0.0635 & $K 1<K 2$ & $0.0079 * * *$ \\
\hline$U 1_{12}$ & 0.0800 & 0.0543 & 0.0830 & 0.0343 & 0.0643 & $U 1>U 2$ & 0.3135 \\
\hline$K 2_{12}$ & 0.0718 & 0.1165 & 0.1265 & 0.0853 & 0.1165 & $K 1<U 1$ & 0.2421 \\
\hline$U 2_{12}$ & 0.0088 & -0.0028 & 0.0705 & 0.1198 & 0.0600 & $K 2>U 2$ & $0.0357 * *$ \\
\hline \multicolumn{8}{|l|}{ Rounds 1-30 } \\
\hline$K 1_{12}$ & 0.0601 & 0.0774 & 0.0670 & 0.0773 & 0.0663 & $K 1<K 2$ & 0.1230 \\
\hline$U 1_{12}$ & 0.0882 & 0.0730 & 0.0831 & 0.0740 & 0.0777 & $U 1<U 2$ & 0.3492 \\
\hline$K 2_{12}$ & 0.0665 & 0.0739 & 0.1091 & 0.0692 & 0.0943 & $K 1<U 1$ & $0.0397 * *$ \\
\hline$U 2_{12}$ & 0.0800 & 0.0223 & 0.0780 & 0.0899 & 0.0768 & $K 2>U 2$ & 0.2262 \\
\hline \multicolumn{8}{|c|}{ Comparison of 8- and 12-subject treatments } \\
\hline$K 1_{8}>K 1_{12}$ & $0.0516^{*}$ & $U 1_{8}>U 1_{12}$ & $0.0873^{*}$ & $K 2_{8}>K 2_{12}$ & $0.004 * * *$ & $U 2_{8}>U 2_{12}$ & $0.004 * * *$ \\
\hline
\end{tabular}

1. The null hypothesis is that average earning is equal in the two treatments.

2. Significant at: * $10 \%$ level; ** 5\% level; *** $1 \%$ level.

Table 9: Bidder Earnings in Early rounds and Over All Rounds

\begin{tabular}{lrrrrrrl}
\hline \hline Treatment & Session 1 & Session 2 & Session 3 & Session 4 & Session 5 & $H_{1}$ & p-value \\
\hline$K 1_{8}$ & 0.8667 & 0.9083 & 0.9167 & 0.9083 & 0.8750 & $K 1>K 2$ & 0.3373 \\
$U 1_{8}$ & 0.8833 & 0.8750 & 0.9000 & 0.8917 & 0.9083 & $U 1>U 2$ & 0.3214 \\
$K 2_{8}$ & 0.8583 & 0.9167 & 0.8917 & 0.8833 & 0.9000 & $K 1>U 1$ & 0.3810 \\
$U 2_{8}$ & 0.9333 & 0.7833 & 0.8250 & 0.9000 & 0.9417 & $K 2>U 2$ & 0.3413 \\
\hline$K 1_{12}$ & 0.6500 & 0.7000 & 0.6583 & 0.7583 & 0.6417 & $K 1<K 2$ & 0.1429 \\
$U 1_{12}$ & 0.7583 & 0.8833 & 0.7417 & 0.7583 & 0.7833 & $U 1>U 2$ & $0.0159 * *$ \\
$K 2_{12}$ & 0.6583 & 0.6917 & 0.8083 & 0.6500 & 0.7917 & $K 1<U 1$ & $0.0040^{* * *}$ \\
$U 2_{12}$ & 0.6333 & 0.5750 & 0.7667 & 0.7083 & 0.6083 & $K 2>U 2$ & 0.1190 \\
\hline \hline Notes: & & & & & &
\end{tabular}

1. The null hypothesis is that efficiency is equal in the two treatments.

2. Significant at: * $10 \%$ level; ** 5\% level; *** $1 \%$ level.

Table 10: Efficiency in 8-subject and 12-subject Treatments and Results of Permutation Tests (one-tailed) 


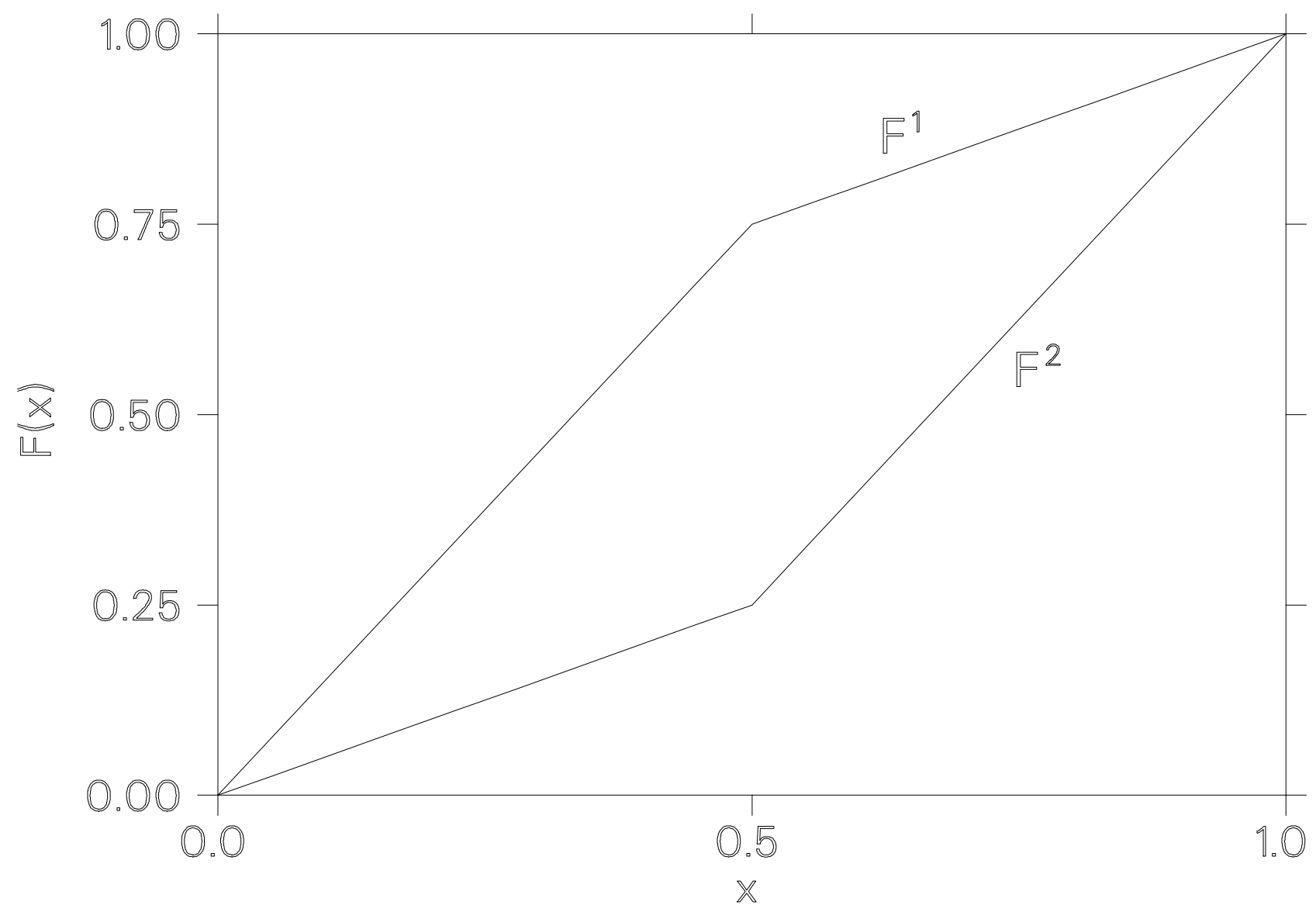

Figure 1: Cumulative Distribution Functions $F^{1}$ and $F^{2}$ 

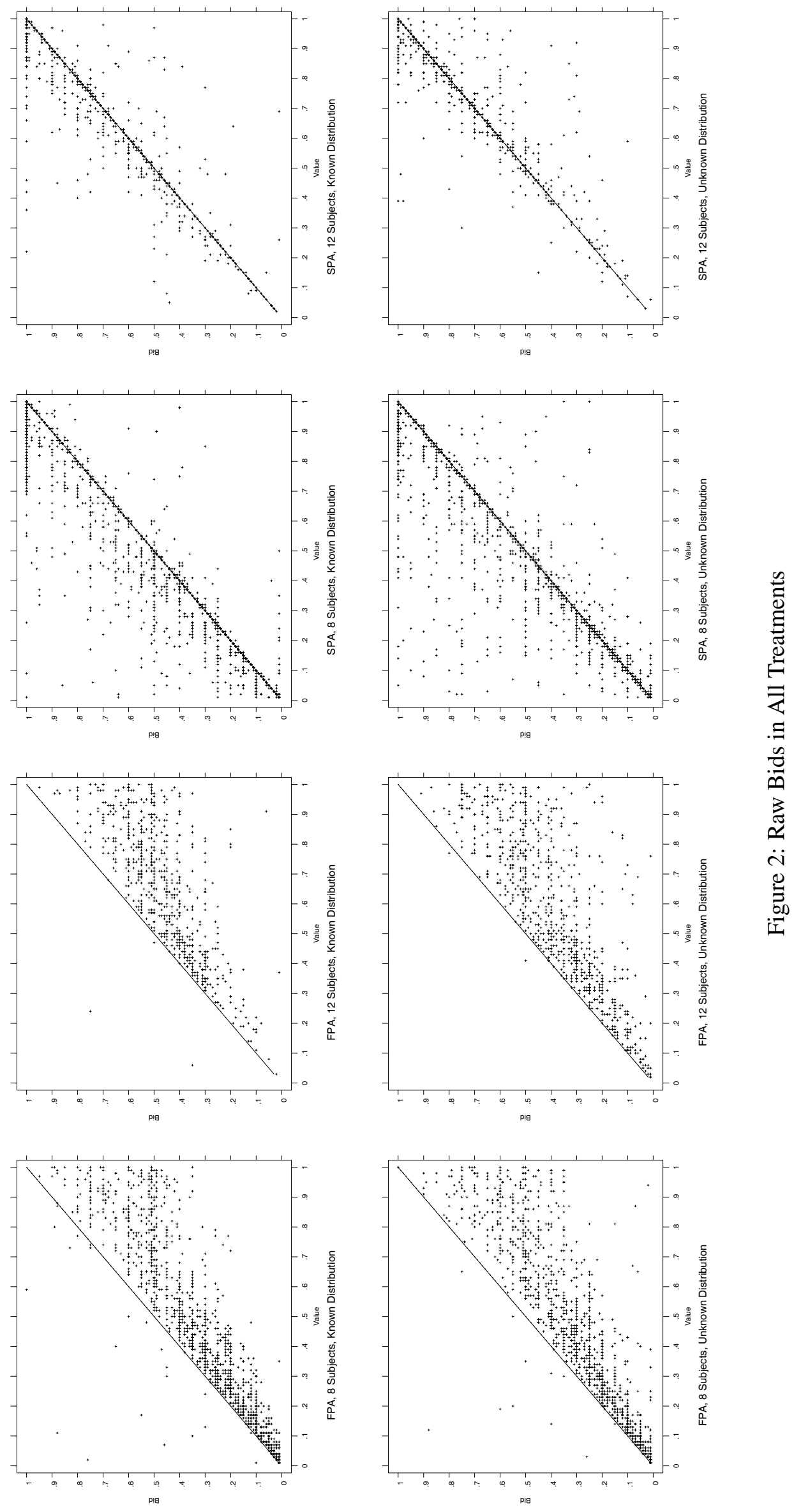

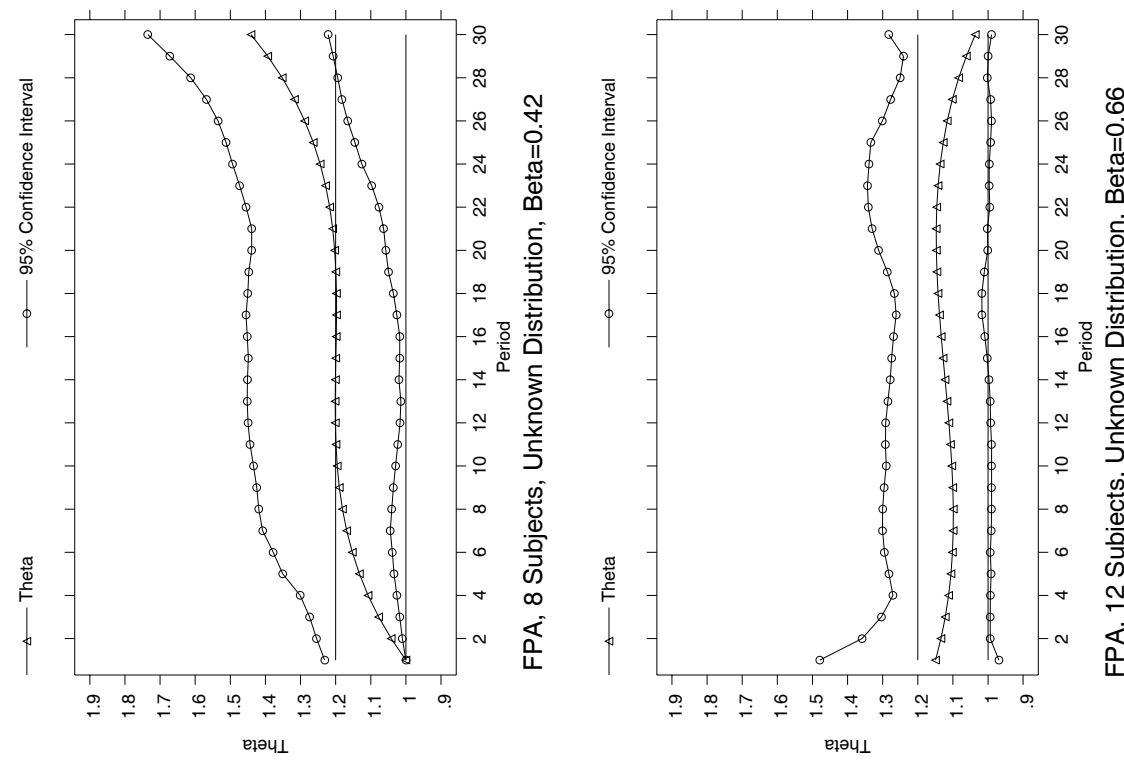

苂
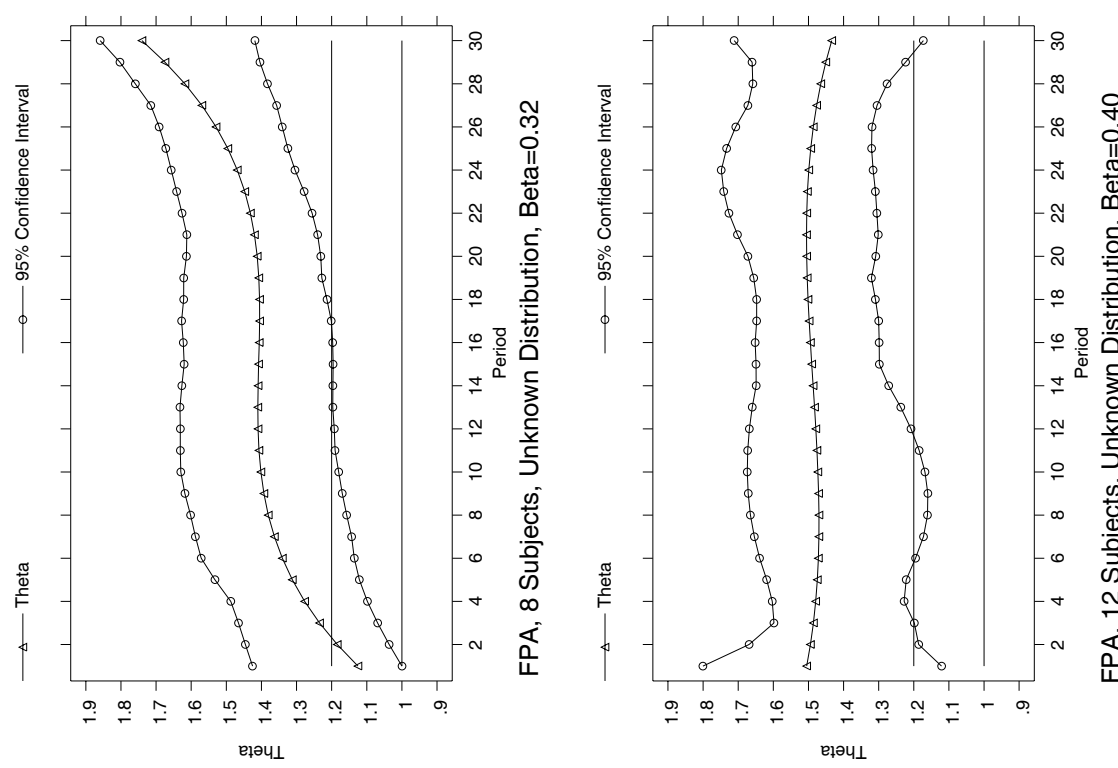

כ)
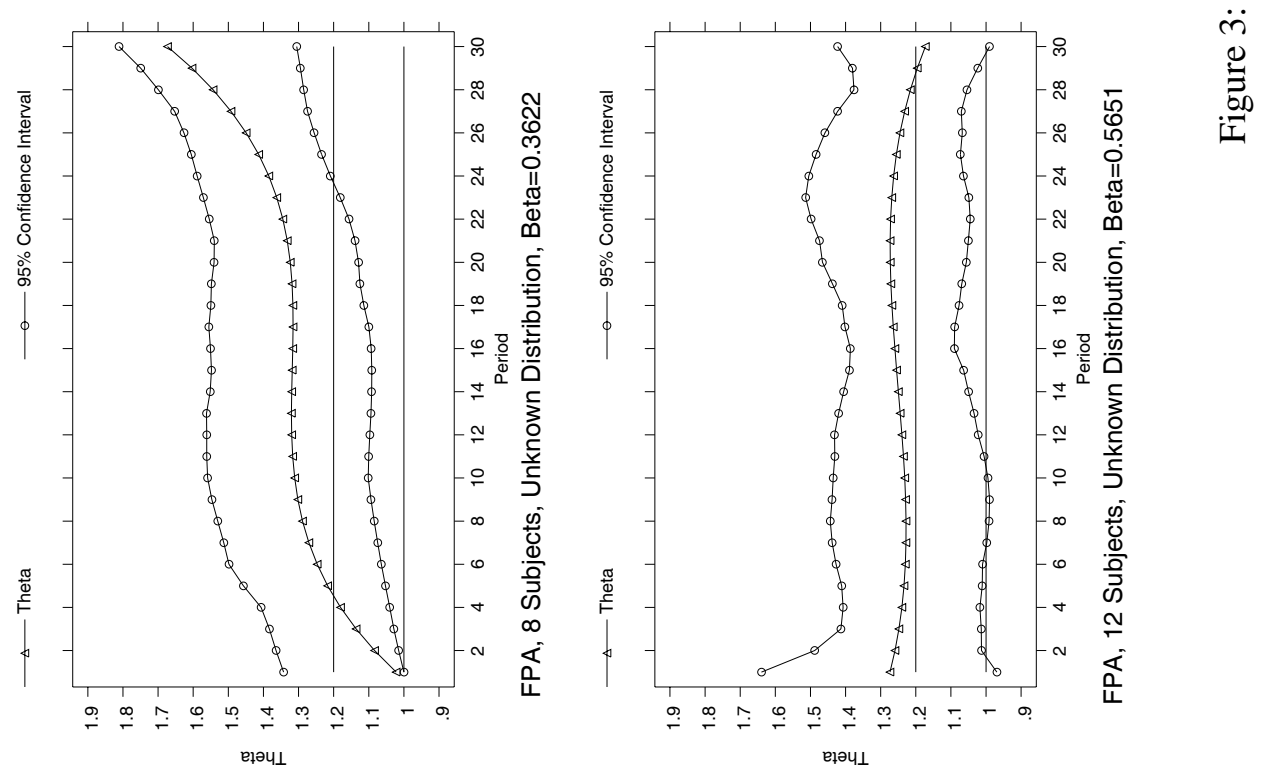

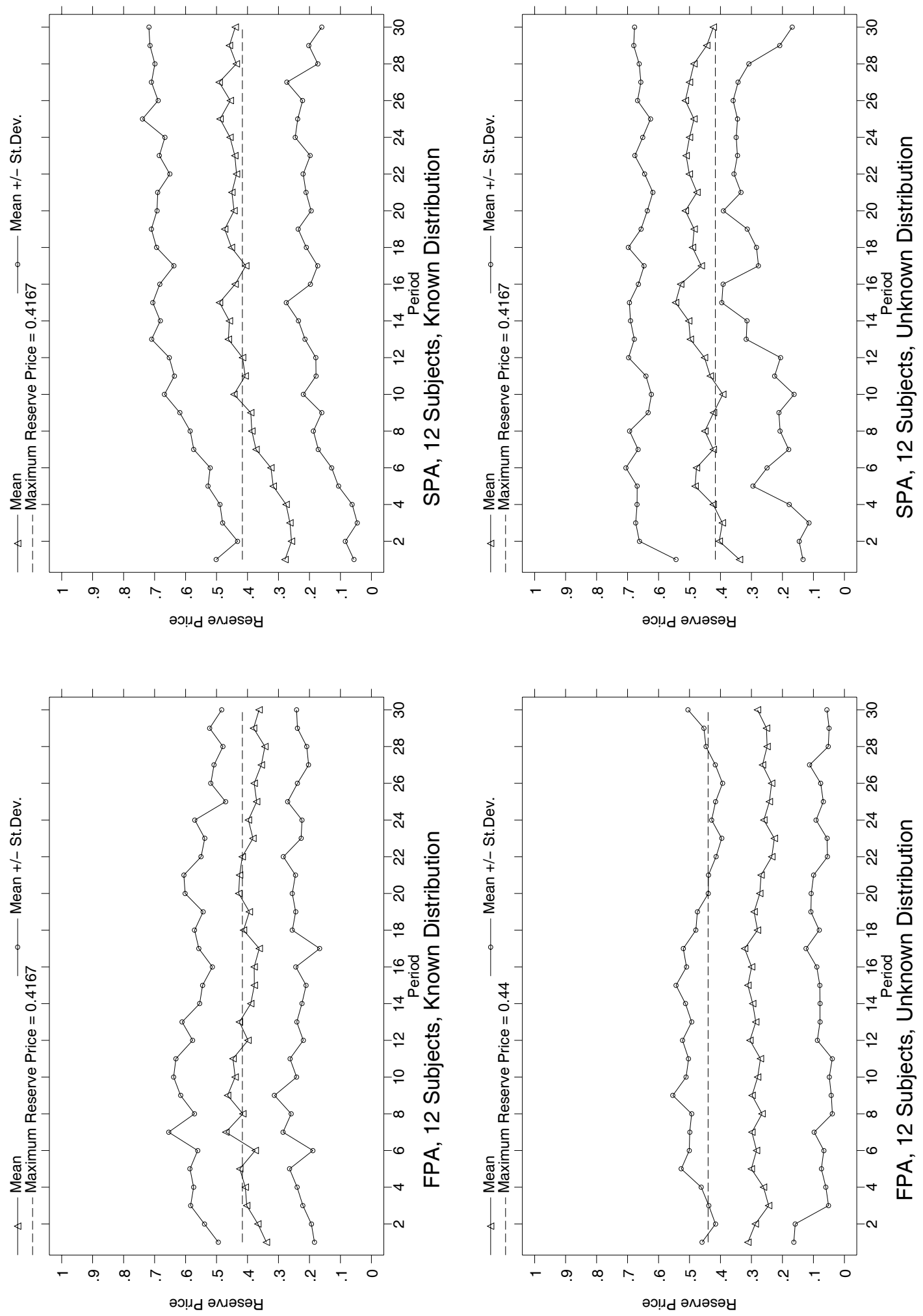

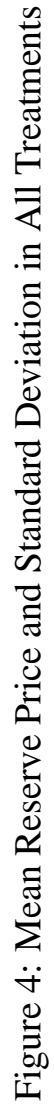

